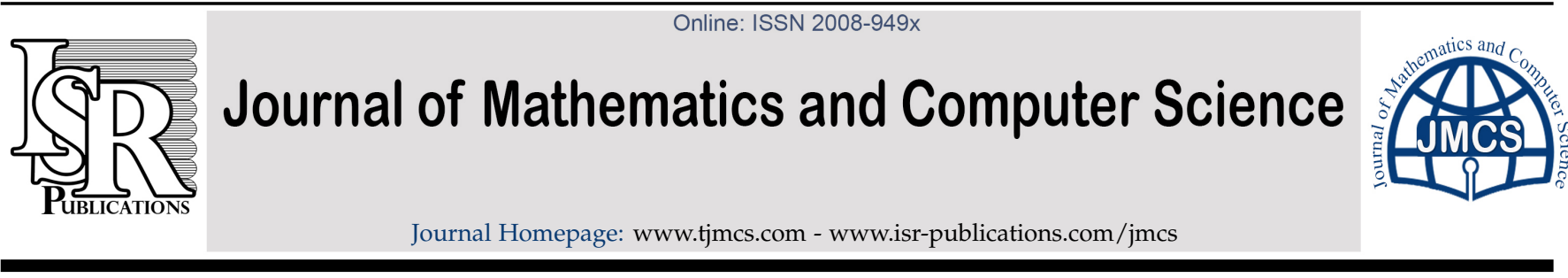

\title{
Results on soft extremally disconnectedness of soft topological spaces
}

\author{
Baravan A. Asaad \\ Department of Mathematics, Faculty of Science, University of Zakho, Kurdistan-region, Iraq.
}

\begin{abstract}
Molodtsov [D. Molodtsov, Global optimization, control, and games, III, Comput. Math. Appl., 37 (1999), 19-31] studied the concept of soft sets. The concept of soft sets is introduced as a general mathematical tool for dealing with uncertainty. In this paper, we give some basic relations about different classes of soft sets and soft closure operator. The purpose of this paper is to introduce soft extremally disconnected spaces via soft sets. Furthermore, some relations of soft sets and soft closure via soft extremally disconnected spaces have been investigated. (C)2017 All rights reserved.
\end{abstract}

Keywords: Soft sets, soft extremally disconnected spaces, soft $\lambda$-open sets where $\lambda \in\{$ regular, $\alpha$, pre, semi, $b, \beta\}$. 2010 MSC: 54G05, 03E75, 54A05.

\section{Introduction and preliminaries}

The concept of soft sets was first constructed by Molodtsov [16] in 1999 as a general mathematical tool for dealing with uncertain objects. He successfully applied the soft set theory in several directions of mathematics, such as smoothness of functions, game theory, operations research, Riemann integration, Perron integration, probability, theory of measurement, and so on.

Maji et al. [14, 15] presented an application of soft sets in decision making problems that is based on the reduction of parameters to keep the optimal choice objects. Chen et al. [5] presented a new definition of soft set parametrization reduction and a comparison of it with attribute reduction in rough set theory. Pei and Miao [17] showed that soft sets are a class of special information systems. Kong et al. [13] introduced the notion of normal parameter reduction of soft sets and its use to investigate the problem of sub-optimal choice and added a parameter set in soft sets. Zou and Xiao [22] discussed the soft data analysis approach.

The soft set theory has been applied to many different fields (see, for example, [8, 12, 17, 21, 22]). Recently, in 2011, Shabir and Naz [18] constituted the study of soft topological spaces. They defined a soft topology on the collection of soft sets over X. Consequently, they defined basic notions of soft topological spaces such as soft open and soft closed sets, soft subspace, soft closure, soft neighbourhood of a point, soft separation axioms, soft regular spaces and soft normal spaces and established several properties for them.

Email address: baravan.asaad@uoz.edu.krd (Baravan A. Asaad)

doi:10.22436/jmcs.017.04.02 
The main purpose of this paper is to introduce soft extremally disconnected topological spaces which are defined over an initial universe with a fixed set of parameters. In Section 2, we give known basic notions and results concerning the theory of soft sets and soft topological spaces. In Section 3, we give some basic relations about different classes of soft sets and soft closure operator. In Section 4, we introduce soft extremally disconnected spaces and we investigate some relations of soft sets and soft closure via the new soft space.

\section{Soft set theory}

Definition 2.1 ([16]). Let $X$ be an initial universe and $E$ be a set of parameters. Let $P(X)$ denote the power set of $X$ and $A$ be a nonempty subset of $E$. A pair $(F, A)$ is called a soft set over $X$, where $F$ is a mapping given by $F: A \rightarrow P(X)$. In other words, a soft set over $X$ is a parametrized family of subsets of the universe $X$. For a particular $e \in A, F(e)$ may be considered the set of e-approximate elements of the soft set $(F, A)$ and if $e \notin A$, then $F(e)=\tilde{\phi}$. The family of all these soft sets over the universal set $X$ is denoted by $\operatorname{SS}(X)_{A}$. Clearly, a soft set is not a set.

Definition $2.2([14])$. For two soft sets $(F, A)$ and $(G, B)$ over a common universe $X$, we say that $(F, A)$ is a soft subset of $(G, B)$ (we write $(F, A) \tilde{\subseteq}(G, B))$ if $A \subseteq B$, and if for all $e \in A, F(e)$ and $G(e)$ are identical approximations. We also say that these two soft sets are soft equal if $(F, A)$ is a soft subset of $(G, B)$ and $(G, B)$ is a soft subset of $(F, A)$.

Definition 2.3 ([14]). A soft set $(F, A)$ over $X$ is said to be:

1. A null soft set, denoted by $\tilde{\phi}$ if for all $\epsilon \in A, F(\epsilon)=\phi$.

2. An absolute soft set, denoted by $\tilde{A}$ if for all $\epsilon \in A, F(\epsilon)=X$.

Definition 2.4 ([14]). The complement of a soft set $(F, A)$ is denoted by $(F, A)^{c}$ and is defined by $(F, A)^{c}=$ $\left(F^{c}, A\right)$ where $F^{c}: A \rightarrow P(X)$ is a mapping given by $F^{c}(\epsilon)=X \backslash F(\epsilon)$ for all $\epsilon \in A$.

Definition 2.5 ([14]). The soft union of two soft sets $(F, A)$ and $(G, B)$ over the common universe $X$ is the soft set $(H, C)$, where $C=A \cup B$, denoted by $(H, C)=(F, A) \tilde{\cup}(G, B)$, and is defined as for all $e \in C$,

$$
H(e)= \begin{cases}F(e), & \text { if } e \in A \backslash B, \\ G(e), & \text { if } e \in B \backslash A, \\ F(e) \cup G(e), & \text { if } e \in A \cap B .\end{cases}
$$

Definition 2.6 ([7]). The soft intersection of two soft sets $(F, A)$ and $(G, B)$ over a common universe $X$ is the soft set $(H, C)$ where $C=A \cap B$, denoted by $(H, C)=(F, A) \tilde{\cap}(G, B)$, and is defined as $H(e)=F(e) \cap G(e)$ for all $e \in C$.

Definition 2.7 ([18]). The soft difference of two soft sets $(F, A)$ and $(G, A)$ over $X$ is the soft set $(H, A)$, denoted by $(H, A)=(F, A) \widetilde{\backslash}(G, A)$, and is defined as $H(e)=F(e) \backslash G(e)$ for all $e \in E$.

Definition $2.8([6])$. A soft set $(F, A)$ over $X$ is said to be a soft point if there exists exactly one $\alpha \in A$, such that $F(\alpha)=\{x\}$ for some $x \in X$ and $F(\beta)=\phi$, for all $\beta \in A \backslash\{\alpha\}$. It will be denoted by $x_{\alpha}$. A soft point $x_{\alpha}$ belongs to a soft set $(F, A)$ if $x \in F(\alpha)$ and it is denoted by $x_{\alpha} \in(F, A)$. Two soft points $x_{\alpha}, y_{\beta}$ are said to be equal if $\alpha=\beta$ and $F(\alpha)=F(\beta)$. Thus $x_{\alpha} \neq y_{\beta}$ if and only if $\alpha \neq \beta$ or $F(\alpha) \neq F(\beta)$.

Definition 2.9 ([18]). Let $\tau$ be the collection of soft sets over $X$, then $\tau$ is said to be a soft topology on $X$ if it satises the following axioms

1. $\tilde{\phi}, \tilde{X}$ belong to $\tau$.

2. The union of any number of soft sets in $\tau$ belongs to $\tau$.

3. The intersection of any two soft sets in $\tau$ belongs to $\tau$. 
The triple $(X, \tau, A)$ is said to be a soft topological space (or soft space) over $X$. Every member of $\tau$ is called a soft open set in $X$. The complement of any soft open set in $X$ is called a soft closed set in $X$. Arbitrary soft union of soft open sets is soft open and finite soft intersection of soft closed sets is soft closed.

Definition 2.10. Let $(X, \tau, A)$ be a soft topological space and let $(F, A)$ be a soft set over $X$. Then

1. The soft closure of $(F, A)$ is the soft set defined by

$$
\tilde{\operatorname{scl}}(F, A)=\tilde{\cap}\{(G, A):(G, A) \text { is a soft closed set and }(G, A) \tilde{\subseteq}(F, A)\} .
$$

Clearly $\tilde{\operatorname{scl}}(\mathrm{F}, \mathrm{A})$ is the smallest soft closed set over $X$ which contains $(F, A)[18]$.

2. The soft interior of $(F, A)$ is the soft set defined by

$$
\tilde{\operatorname{sint}}(F, A)=\tilde{\cup}\{(G, A):(G, A) \text { is a soft open set and }(F, A) \tilde{\subseteq}(G, A)\} .
$$

Thus $\tilde{\operatorname{sint}}(F, A)$ is the largest soft open set contained in $(F, A)[9]$.

Lemma 2.11 ([9]). Let $(F, A)$ be any soft subset of a soft topological space $(X, \tau, A)$. Then

$$
\tilde{\operatorname{scl}}(F, A)=X \tilde{\operatorname{sint}}(X \tilde{\backslash}(F, A)) \text { and } \tilde{\operatorname{sint}}(F, A)=X \tilde{\Upsilon} \tilde{\operatorname{scl}}(X \tilde{\backslash}(F, A)) \text {. }
$$

Lemma 2.12 ([20]). Let $(\mathrm{X}, \tau, \mathrm{A})$ be a soft topological space. Then for every soft open set $(\mathrm{U}, \mathrm{A})$ and every soft subset $(F, A)$ of $X$, we have $\tilde{\operatorname{scl}}(F, A) \tilde{\cap}(U, A) \tilde{\subseteq} \tilde{\operatorname{scl}}((F, A) \tilde{\cap}(U, A))$.

Definition 2.13. A soft subset $(F, A)$ of a soft topological space $(X, \tau, A)$ is said to be:

1. soft regular-open if $(F, A)=\tilde{\operatorname{sint}}(\tilde{\operatorname{scl}}(F, A))[19] ;$

2. soft $\alpha$-open if $(F, A) \tilde{\subseteq} \tilde{\operatorname{sint}}(\tilde{\operatorname{scl}}(\tilde{\operatorname{sint}}(F, A)))[1]$;

3. soft semiopen if $(F, A) \tilde{\subseteq} \tilde{\operatorname{scl}}(\tilde{\operatorname{sint}}(F, A))[4] ;$

4. soft preopen if $(F, A) \widetilde{\subseteq} \operatorname{sint}(\tilde{\operatorname{scl}}(F, A))[10]$;

5. soft b-open if $(F, A) \tilde{\subseteq} \tilde{\operatorname{scl}}(\tilde{\operatorname{sint}}(F, A)) \tilde{\cup} \tilde{\operatorname{sint}}(\tilde{\operatorname{scl}}(F, A))[2]$;

6. soft $\beta$-open if $(F, A) \simeq \tilde{\operatorname{scl}}(\tilde{\operatorname{sint}}(\tilde{\operatorname{scl}}(F, A)))[3]$.

Definition 2.14. The complement of a soft regular-open (resp. soft $\alpha$-open, soft semiopen, soft preopen, soft b-open and soft $\beta$-open) set is said to be soft regular-closed [19] (resp. soft $\alpha$-closed [1], soft semiclosed [4], soft preclosed [10], soft b-closed [2] and soft $\beta$-closed [3]).

Definition 2.15. For any soft topological space $(X, \tau, A)$, we denote the family of all soft regular-open (resp. soft $\alpha$-open, soft semiopen, soft preopen, soft $b$-open, soft $\beta$-open, soft regular-closed, soft closed, soft semiclosed, soft preclosed, soft b-closed and soft $\beta$-closed) sets of a soft space $X$ by $S R O(X)$ (resp. $\mathrm{S} \alpha \mathrm{O}(X), \operatorname{SPO}(X), \operatorname{SSO}(X), \operatorname{SBO}(X), \operatorname{S} \beta O(X), \operatorname{SRC}(X), \operatorname{SC}(X), \operatorname{SPC}(X), \operatorname{SSC}(X), \operatorname{SBC}(X)$ and $\operatorname{S} \beta C(X))$.

Definition 2.16. Let $(X, \tau, A)$ be a soft topological space and let $(F, A) \in \operatorname{SS}(X)_{A}$. Then the soft $\alpha$-closure [1] (resp. soft preclosure [10], soft semiclosure [4], soft b-closure [2] and soft $\beta$-closure [3]) of $(F, A)$ is the soft intersection of all soft $\alpha$-closed (resp. soft preclosed, soft semiclosed, soft b-closed and soft $\beta$-closed) sets of $(X, \tau, A)$ containing $(F, A)$ and is denoted by $\tilde{\operatorname{s}} \alpha c l(F, A)(\operatorname{resp} . \tilde{s} p c l(F, A), \tilde{s} s c l(F, A), \tilde{s} b c l(F, A)$ and $\tilde{s} \beta c l(F, A))$.

Definition 2.17. Let $(X, \tau, A)$ be a soft topological space and let $(F, A) \in S S(X)_{A}$. Then the soft $\alpha$-interior [1] (resp. soft preinterior [10], soft semiinterior [4], soft b-interior [2] and soft $\beta$-interior [3]) of $(F, A)$ is the soft union of all soft $\alpha$-open (resp. soft preopen, soft semiopen, soft b-open and soft $\beta$-open) sets of $X$ contained in $(F, A)$ and is denoted by $\tilde{s} \alpha \operatorname{int}(F, A)(\operatorname{resp} . \tilde{s} \operatorname{pint}(F, A), \tilde{\sin } \operatorname{sint}(F, A), \tilde{s} b i n t(F, A)$ and $\tilde{s} \beta \operatorname{int}(F, A))$. 
Theorem 2.18. Let $(F, A)$ be any soft subset of a soft topological space $(X, \tau, A)$. Then the following statements hold:

1. $\tilde{\operatorname{spcl}}(F, A)=(F, A) \tilde{\cup} \tilde{\operatorname{scl}}(\tilde{\operatorname{sint}}(F, A))[10]$.

2. $\tilde{\operatorname{sscl}}(F, A)=(F, A) \tilde{\cup} \tilde{\operatorname{sint}}(\tilde{\operatorname{scl}}(F, A))[4]$.

3. $\tilde{\operatorname{sbcl}}(F, A)=\tilde{\operatorname{spcl}}(F, A) \tilde{\cap} \tilde{\operatorname{sscl}}(F, A)[2]$.

4. $\tilde{s} \beta \operatorname{cl}(F, A)=(F, A) \tilde{\cup} \tilde{\operatorname{sint}}(\tilde{\operatorname{scl}}(\tilde{\operatorname{sint}}(F, A)))[3]$.

Theorem 2.19 ([20]). For any soft subset $(F, A)$ of a soft topological space $(X, \tau, A)$, then $(F, A) \tilde{\in} S \beta O(X)$ if and only if $\tilde{\operatorname{scl}}(\mathrm{F}, \mathrm{A}) \tilde{\in} \mathrm{SRC}(\mathrm{X})$.

Some examples of soft topological spaces are as follows.

Example 2.20. Consider the space $X=\left\{a_{1}, a_{2}, a_{3}\right\}$ and $A=\left\{e_{1}, e_{2}\right\}$ with the topology

$$
\tau=\left\{\tilde{\phi}, \tilde{X},\left(F_{1}, A\right),\left(F_{2}, A\right),\left(F_{3}, A\right),\left(F_{4}, A\right),\left(F_{5}, A\right),\left(F_{6}, A\right),\left(F_{7}, A\right)\right\},
$$

where $\left(F_{1}, A\right),\left(F_{2}, A\right),\left(F_{3}, A\right),\left(F_{4}, A\right),\left(F_{5}, A\right),\left(F_{6}, A\right)$ and $\left(F_{7}, A\right)$ are soft sets over $X$, defined as follows:

$$
\begin{aligned}
& F_{1}\left(e_{1}\right)=\left\{a_{1}, a_{2}\right\}, F_{1}\left(e_{2}\right)=\left\{a_{1}, a_{2}\right\}, \\
& F_{2}\left(e_{1}\right)=\left\{a_{2}\right\}, F_{2}\left(e_{2}\right)=\left\{a_{1}, a_{3}\right\}, \\
& F_{3}\left(e_{1}\right)=\left\{a_{2}, a_{3}\right\}, F_{3}\left(e_{2}\right)=\left\{a_{1}\right\}, \\
& F 4\left(e_{1}\right)=\left\{a_{2}\right\}, F_{4}\left(e_{2}\right)=\left\{a_{1}\right\}, \\
& F_{5}\left(e_{1}\right)=\left\{a_{1}, a_{2}\right\}, F_{5}\left(e_{2}\right)=X, \\
& F_{6}\left(e_{1}\right)=X, F_{6}\left(e_{2}\right)=\left\{a_{1}, a_{2}\right\}, \\
& F_{7}\left(e_{1}\right)=\left\{a_{2}, a_{3}\right\}, F_{7}\left(e_{2}\right)=\left\{a_{1}, a_{3}\right\} .
\end{aligned}
$$

Then $\tau$ defines a soft topology on $X$. Hence $(X, \tau, A)$ is a soft topological space over $X$. The soft sets $\left(G_{1}, A\right)$ and $\left(G_{2}, A\right)$ in $(X, \tau, A)$ are defined as follows:

$$
\begin{aligned}
& \mathrm{G}_{1}\left(e_{1}\right)=\left\{\mathrm{a}_{2}, \mathrm{a}_{3}\right\}, \mathrm{G}_{1}\left(\mathrm{e}_{2}\right)=\left\{\mathrm{a}_{1}, \mathrm{a}_{2}\right\}, \\
& \mathrm{G}_{2}\left(\mathrm{e}_{1}\right)=\phi, \mathrm{G}_{2}\left(e_{2}\right)=\left\{\mathrm{a}_{1}\right\} .
\end{aligned}
$$

Then the two soft sets $\left(G_{1}, A\right)$ and $\left(G_{2}, A\right)$ are not soft open since $\left(G_{1}, A\right) \notin \tau$ and $\left(G_{2}, A\right) \notin \tau$.

Example 2.21. Let $X=\left\{a_{1}, a_{2}, a_{3}, a_{4}\right\}$ and $A=\left\{e_{1}, e_{2}\right\}$ with the topology $\tau=\left\{\tilde{\phi}, \tilde{X},\left(F_{1}, A\right),\left(F_{2}, A\right),\left(F_{3}, A\right)\right\}$ where $\left(F_{1}, A\right),\left(F_{2}, A\right)$ and $\left(F_{3}, A\right)$ are soft sets over $X$, which is defined as follows:

$$
\begin{aligned}
& F_{1}\left(e_{1}\right)=\left\{a_{1}, a_{3}\right\}, F_{1}\left(e_{2}\right)=\phi, \\
& F_{2}\left(e_{1}\right)=\left\{a_{4}\right\}, F_{2}\left(e_{2}\right)=\left\{a_{4}\right\}, \\
& F_{3}\left(e_{1}\right)=\left\{a_{1}, a_{3}, a_{4}\right\}, F_{3}\left(e_{2}\right)=\left\{a_{4}\right\} .
\end{aligned}
$$

Then $\tau$ defines a soft topology on $X$ and hence $(X, \tau, A)$ is a soft topological space over $X$. The soft sets $\left(G_{1}, A\right)$ and $\left(G_{2}, A\right)$ in $(X, \tau, A)$ are defined as follows:

$$
\begin{aligned}
& \mathrm{G}_{1}\left(e_{1}\right)=\left\{\mathrm{a}_{2}, \mathrm{a}_{3}\right\}, \mathrm{G}_{1}\left(e_{2}\right)=\left\{\mathrm{a}_{3}\right\}, \\
& \mathrm{G}_{2}\left(e_{1}\right)=\left\{\mathrm{a}_{2}, \mathrm{a}_{4}\right\}, \mathrm{G}_{2}\left(e_{2}\right)=\left\{\mathrm{a}_{1}, \mathrm{a}_{4}\right\} .
\end{aligned}
$$

Then the two soft sets $\left(G_{1}, A\right)$ and $\left(G_{2}, A\right)$ are not soft open since $\left(G_{1}, A\right) \notin \tau$ and $\left(G_{2}, A\right) \notin \tau$.

Example 2.22. Let $X=\left\{a_{1}, a_{2}, a_{3}, a_{4}\right\}, A=\left\{e_{1}, e_{2}\right\}$ and $\tau=\left\{\tilde{\phi}, \tilde{X},\left(F_{1}, A\right),\left(F_{2}, A\right),\left(F_{3}, A\right)\right\}$ where $\left(F_{1}, A\right)$, $\left(F_{2}, A\right)$ and $\left(F_{3}, A\right)$ are soft sets over $X$, which is defined as follows:

$$
\begin{aligned}
& F_{1}\left(e_{1}\right)=\left\{a_{1}\right\}, F_{1}\left(e_{2}\right)=\left\{a_{2}\right\}, \\
& F_{2}\left(e_{1}\right)=\left\{a_{1}, a_{2}\right\}, F_{2}\left(e_{2}\right)=\left\{a_{2}\right\}, \\
& F_{3}\left(e_{1}\right)=\left\{a_{1}\right\}, F_{3}\left(e_{2}\right)=\left\{a_{1}, a_{2}\right\} .
\end{aligned}
$$

Then $\tau$ defines a soft topology on $X$ and hence $(X, \tau, A)$ is a soft topological space over $X$.

Example 2.23. Let $X=\left\{a_{1}, a_{2}\right\}, A=\left\{e_{1}, e_{2}\right\}$ and $\tau=\left\{\tilde{\phi}, \tilde{X},\left(F_{1}, A\right),\left(F_{2}, A\right),\left(F_{3}, A\right)\right\}$ where $\left(F_{1}, A\right),\left(F_{2}, A\right)$ and $\left(F_{3}, A\right)$ are soft sets over $X$, which is defined as follows:

$$
\begin{aligned}
& F_{1}\left(e_{1}\right)=X, F_{1}\left(e_{2}\right)=\left\{a_{2}\right\}, \\
& F_{2}\left(e_{1}\right)=\left\{a_{1}\right\}, F_{2}\left(e_{2}\right)=X, \\
& F_{3}\left(e_{1}\right)=\left\{a_{1}\right\}, F_{3}\left(e_{2}\right)=\left\{a_{2}\right\} .
\end{aligned}
$$

Then $\tau$ defines a soft topology on $X$ and hence $(X, \tau, A)$ is a soft topological space over $X$. 


\section{Relations on $\tilde{\mathbf{s}} \mathrm{cl}$ operator}

In this section, we obtain more relations on the notion of $\tilde{s} \mathrm{cl}$ operator.

Lemma 3.1. Let $(F, A)$ and $(K, A)$ be any soft subsets of a soft topological space $(X, \tau, A)$. If $(F, A) \tilde{\cap}(K, A)=\tilde{\phi}$, then $\tilde{\operatorname{sint}}(F, A) \tilde{\cap} \tilde{\operatorname{scl}}(K, A)=\tilde{\phi}$ and $\tilde{\operatorname{scl}}(F, A) \tilde{\cap} \tilde{\operatorname{sint}}(K, A)=\tilde{\phi}$.

Proof. It is obvious.

Remark 3.2. Let $(F, A)$ be any soft subset of a soft topological space $(X, \tau, A)$. Then

1. $\tilde{\operatorname{sint}}(F, A) \tilde{\subseteq} \tilde{\operatorname{sint}}(F, A) \tilde{\subseteq} \tilde{\operatorname{spint}}(F, A) \tilde{\subseteq} \tilde{\operatorname{sint}}(F, A) \tilde{\subseteq} \tilde{\operatorname{sint}}(F, A) \tilde{\subseteq}(F, A)$.

2. $\tilde{\operatorname{sint}}(F, A) \tilde{\subseteq} \tilde{\operatorname{sint}}(F, A) \tilde{\subseteq} \operatorname{sint}(F, A) \tilde{\subseteq} \operatorname{sint}(F, A) \tilde{\subseteq} \tilde{\operatorname{sint}}(F, A) \tilde{\subseteq}(F, A)$.

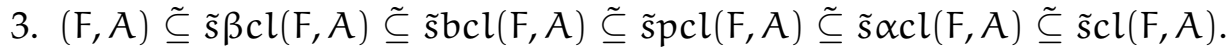

4. $(F, A) \simeq \tilde{s} \beta c l(F, A) \simeq \tilde{s} b c l(F, A) \simeq \tilde{s} \operatorname{scl}(F, A) \tilde{\subseteq} \tilde{s} \alpha c l(F, A) \tilde{\subseteq} \tilde{s c l}(F, A)$.

Lemma 3.3. If $(F, A)$ is any soft $\beta$-open subset of a soft topological space $(X, \tau, A)$, then $\tilde{\operatorname{scl}}(F, A)=\tilde{s} \alpha c l(F, A)$.

Proof. Let $(F, A) \tilde{\in} S \beta O(X)$ and $x_{\alpha} \tilde{\notin} \tilde{s} \alpha c l(F, A)$, then there exists a soft $\alpha$-open set $(U, A)$ containing $x_{\alpha}$ such that $(F, A) \tilde{\cap}(U, A)=\tilde{\phi}$. So by using Lemma 3.1, we have

$$
\tilde{\sin } \operatorname{cl}(\tilde{\operatorname{sint}}(\tilde{\operatorname{scl}}(F, A))) \tilde{\cap} \tilde{\operatorname{sint}}(\tilde{\operatorname{scl}}(\tilde{\operatorname{sint}}(U, A)))=\tilde{\phi} .
$$

Since $(F, A)$ is soft $\beta$-open, then $(F, A) \tilde{\cap} \tilde{\operatorname{sint}}(\tilde{\operatorname{scl}}(\tilde{\operatorname{sint}}(U, A)))=\tilde{\phi}$. Since $(U, A)$ is soft $\alpha$-open set containing $x_{\alpha}$, then $x_{\alpha} \tilde{\in} \tilde{\operatorname{sint}}(\tilde{\operatorname{scl}}(\tilde{\operatorname{sint}}(\mathrm{U}, A)))$ and $\tilde{\operatorname{sint}}(\tilde{\operatorname{scl}}(\tilde{\operatorname{sint}}(\mathrm{U}, A)))$ is soft open set. So $x_{\alpha} \tilde{\notin} \tilde{\operatorname{scl}}(\mathrm{F}, A)$. Hence $\tilde{\operatorname{scl}}(F, A) \tilde{\simeq} \tilde{\operatorname{s}} \alpha c l(F, A)$. But $\tilde{\operatorname{sicl}} \alpha(F, A) \tilde{\simeq} \tilde{\operatorname{scl}}(F, A)$ in general. Thus, $\tilde{\operatorname{scl}}(F, A)=\tilde{\operatorname{s}} \alpha \operatorname{cl}(F, A)$.

Lemma 3.4. If $(F, A)$ is any soft preopen subset of a soft topological space $(X, \tau, A)$, then $\tilde{\operatorname{scl}}(F, A)=\tilde{\operatorname{s}} \alpha \mathrm{cl}(F, A)$.

Proof. This is shown in Lemma 3.3 by the fact that every soft preopen set is soft $\beta$-open.

Lemma 3.5 ([11]). For any soft subset $(F, A)$ of a soft space $(X, \tau, A)$, we have

$$
(F, A) \tilde{\in} \operatorname{SSO}(X) \text { if and only if } \tilde{\operatorname{scl}}(\mathrm{F}, \mathrm{A})=\tilde{\operatorname{scl}}(\tilde{\operatorname{sint}}(\mathrm{F}, \mathrm{A})) \text {. }
$$

Lemma 3.6. If $(F, A)$ is any soft semiopen subset of a soft topological space $(X, \tau, A)$, then $\tilde{s} \operatorname{cl}(F, A)=\tilde{s} p c l(F, A)$.

Proof. By (1) of Theorem 2.18 and Lemma 3.5, we have

$$
\tilde{\operatorname{spcl}}(F, A)=(F, A) \tilde{\cup} \tilde{\operatorname{scl}}(\tilde{\operatorname{sint}}(F, A))=(F, A) \tilde{\cup} \tilde{\operatorname{scl}}(F, A)=\tilde{\operatorname{scl}}(F, A) .
$$

Therefore, $\tilde{s} c l(F, A)=\tilde{s} p c l(F, A)$.

By using (3) of Remark 3.2 with Lemma 3.6, we have the following corollary.

Corollary 3.7. For each soft semiopen set $(F, A)$ in $(X, \tau, A)$, we have

$$
\tilde{\operatorname{scl}}(F, A)=\tilde{\operatorname{s}} \alpha \operatorname{cl}(F, A)=\tilde{\operatorname{spcl}}(F, A) .
$$

Lemma 3.8. Let $(F, A)$ be a soft subset of a soft space $(X, \tau, A)$. Then the following statements hold:

1. $\tilde{\operatorname{sscl}}(F, A)=\tilde{\operatorname{sbcl}}(F, A)=\tilde{s} \beta \operatorname{cl}(F, A)$ for each $(F, A) \tilde{\epsilon} \operatorname{SSO}(X)$.

2. $\tilde{\operatorname{sint}}(\mathrm{F}, A)=\tilde{\operatorname{sbint}}(\mathrm{F}, \mathrm{A})=\tilde{\operatorname{s}} \beta \operatorname{int}(\mathrm{F}, A)$ for each $(F, A) \tilde{\in} \operatorname{SSC}(X)$.

Proof.

(1): Let $(F, A) \tilde{\in S S O}(X)$, then by applying Lemma 3.5 and (1), (2) and (3) of Theorem 2.18, we obtain that 


$$
\begin{aligned}
\tilde{\operatorname{sbcl}}(F, A) & =\tilde{\operatorname{spcl}}(F, A) \tilde{\cap} \tilde{\operatorname{scl}}(F, A) \\
& =[(F, A) \tilde{\cup} \tilde{\operatorname{scl}}(\tilde{\operatorname{sint}}(F, A))] \tilde{\cap}[(F, A) \tilde{\cup} \tilde{\operatorname{sint}}(\tilde{\operatorname{scl}}(F, A))] \\
& =(F, A) \tilde{\cup}[\tilde{\operatorname{scl}}(\tilde{\operatorname{sint}}(F, A)) \tilde{\cap} \tilde{\operatorname{sint}}(\tilde{\operatorname{s}} \operatorname{cl}(F, A))] \\
& =(F, A) \tilde{\cup}[\tilde{\operatorname{scl}}(F, A) \tilde{\cap} \tilde{\operatorname{sint}}(\tilde{\operatorname{scl}}(F, A))] \\
& =(F, A) \tilde{\cup} \tilde{\operatorname{sint}}(\tilde{\operatorname{scl}}(F, A))=\tilde{\operatorname{sscl}}(F, A) .
\end{aligned}
$$

On the other hand

$$
\begin{aligned}
\tilde{\operatorname{sbcl}}(F, A) & =(F, A) \tilde{\cup}[\tilde{\operatorname{s}} \operatorname{cl}(\tilde{\operatorname{sint}}(F, A)) \tilde{\operatorname{sint}}(\tilde{\operatorname{scl}}(F, A))] \\
& =(F, A) \tilde{\cup}[\tilde{\operatorname{s}} \operatorname{cl}(\tilde{\operatorname{sint}}(F, A)) \tilde{\cap} \tilde{\operatorname{sint}}(\tilde{\operatorname{scl}}(\tilde{\operatorname{sint}}(F, A)))] \\
& =(F, A) \tilde{\cup} \tilde{\operatorname{sint}}(\tilde{\operatorname{s}} \operatorname{cl}(\tilde{\operatorname{sint}}(F, A)))=\tilde{\operatorname{s}} \beta \operatorname{cl}(F, A),
\end{aligned}
$$

which completes the proof.

(2): It follows immediately by (1).

Lemma 3.9. Let $(F, A)$ be any soft subset of a soft topological space $(X, \tau, A)$, then $(F, A) \tilde{\in} S \beta O(X)$ if and only if $\tilde{\operatorname{scl}}(F, A)=\tilde{\operatorname{scl}}(\tilde{\operatorname{sint}}(\tilde{\operatorname{scl}}(F, A)))$.

Proof. Let $(F, A) \tilde{\in} S \beta O(X)$, then $(F, A) \tilde{\subseteq} \tilde{\operatorname{scl}}(\tilde{\operatorname{sint}}(\tilde{\operatorname{scl}}(F, A)))$ implies that

$$
\tilde{\operatorname{scl}}(F, A) \tilde{\subseteq} \operatorname{scl}(\tilde{\operatorname{sint}}(\tilde{\operatorname{scl}}(F, A))) \widetilde{\operatorname{scl}}(F, A) .
$$

Therefore, $\tilde{\operatorname{scl}}(F, A)=\tilde{\operatorname{scl}}(\tilde{\operatorname{sint}}(\tilde{\operatorname{scl}}(F, A)))$.

Conversely, suppose that $\tilde{\operatorname{scl}}(F, A)=\tilde{\operatorname{scl}}(\tilde{\operatorname{sint}}(\tilde{\operatorname{scl}}(\mathrm{F}, A)))$. This implies that

$$
\tilde{\operatorname{scl}}(F, A) \tilde{\simeq} \tilde{\operatorname{scl}}(\tilde{\operatorname{sint}}(\tilde{\operatorname{scl}}(F, A))) .
$$

Then

$$
(F, A) \simeq \tilde{\operatorname{scl}}(F, A) \tilde{\subseteq} \operatorname{scl}(\tilde{\operatorname{sint}}(\tilde{\operatorname{scl}}(F, A))) .
$$

Hence $(F, A) \tilde{\subseteq} \tilde{\operatorname{scl}}(\tilde{\operatorname{sint}}(\tilde{\operatorname{scl}}(F, A)))$. Therefore, $(F, A) \tilde{\in} S \beta O(X)$.

Lemma 3.10. For any soft subset $(F, A)$ of a soft topological space $(X, \tau, A)$, then

1. $\tilde{\operatorname{scl}}(\tilde{\operatorname{sint}}(\tilde{\operatorname{scl}}(\tilde{\operatorname{sint}}(F, A))))=\tilde{\operatorname{scl}}(\tilde{\operatorname{sint}}(F, A))$.

2. $\tilde{\operatorname{sint}}(\tilde{\operatorname{scl}}(\tilde{\operatorname{sint}}(\tilde{\operatorname{scl}}(F, A))))=\tilde{\operatorname{sint}}(\tilde{\operatorname{scl}}(F, A))$.

Proof.

(1): Since $\tilde{\operatorname{sint}}(\tilde{\operatorname{scl}}(\tilde{\operatorname{sint}}(F, A))) \tilde{\subseteq} \tilde{\operatorname{scl}}(\tilde{\operatorname{sint}}(F, A))$, then

$$
\tilde{\operatorname{scl}} \operatorname{cosint}(\tilde{\operatorname{scl}}(\tilde{\operatorname{sint}}(F, A)))) \tilde{\subseteq} \tilde{\operatorname{scl}}(\tilde{\operatorname{sint}}(F, A)) .
$$

On the other hand, $\operatorname{since} \tilde{\operatorname{sint}}(F, A) \tilde{\subseteq} \tilde{\operatorname{scl}}(\tilde{\operatorname{sint}}(F, A)) \operatorname{implies}$ that $\tilde{\operatorname{sint}}(F, A) \tilde{\subseteq} \operatorname{sint}(\tilde{\operatorname{scl}}(\tilde{\operatorname{sint}}(F, A)))$ and hence $\tilde{\operatorname{scl}}(\tilde{\operatorname{sint}}(F, A)) \simeq \tilde{\operatorname{scl}}(\tilde{\operatorname{sint}}(\tilde{\operatorname{scl}}(\tilde{\operatorname{sint}}(F, A))))$. Therefore, $\tilde{\operatorname{scl}}(\tilde{\operatorname{sint}}(\tilde{\operatorname{scl}}(\tilde{\operatorname{sint}}(F, A))))=\tilde{\operatorname{scl}}(\tilde{\operatorname{sint}}(F, A))$.

(2): The proof is similar to (1).

Theorem 3.11. Let $(F, A)$ be any soft subset of a soft topological space $(X, \tau, A)$. Then

1. $(F, A) \tilde{\in} \operatorname{S} \beta O(X)$ if and only if $\tilde{s} c l(F, A) \tilde{\in} \operatorname{SSO}(X)$.

2. $(F, A) \tilde{\in} S \beta O(X)$ if and only if $\tilde{s} c l(F, A) \tilde{\in} S \beta O(X)$.

3. $(F, A) \tilde{\in} S \beta O(X)$ if and only if $\tilde{\operatorname{scl}}(F, A) \tilde{\in} \operatorname{SBO}(X)$. 
Proof.

(1): Let $(F, A) \tilde{\in} \operatorname{S} \beta O(X)$, then by Theorem 2.19, $\tilde{s} c l(F, A) \tilde{\in} \operatorname{SRC}(X) \tilde{\subseteq} \operatorname{SSO}(X)$. So $\tilde{s} c l(F, A) \tilde{\epsilon} \operatorname{SSO}(X)$. Conversely, let $\tilde{\operatorname{scl}}(F, A) \tilde{\in} \operatorname{SSO}(X)$. Then by Lemma 3.5, $\tilde{\operatorname{scl}}(\tilde{\operatorname{scl}}(F, A))=\tilde{\operatorname{scl}}(\tilde{\operatorname{sint}}(\tilde{\operatorname{scl}}(F, A)))$ which implies that $\tilde{s} c l(F, A)=\tilde{\operatorname{scl}}(\tilde{\operatorname{sint}}(\tilde{\operatorname{scl}}(F, A)))$ and hence by Lemma 3.9, $(F, A) \tilde{\in} \operatorname{S} \beta O(X)$.

(2): Let $(F, A) \tilde{\epsilon} S \beta O(X)$, then by (1), $\tilde{\operatorname{scl}}(F, A) \tilde{\epsilon} S S O(X) \tilde{S} \beta O(X)$. So $\tilde{s} c l(F, A) \tilde{\epsilon} S \beta O(X)$. Conversely, let $\tilde{s} c l(F, A) \tilde{\epsilon} \operatorname{S} \beta O(X)$, by $(1), \tilde{\operatorname{scl}}(\tilde{\operatorname{scl}}(F, A)) \tilde{\epsilon} \operatorname{SSO}(X)$. Since $\tilde{s} \operatorname{cl}(\tilde{\operatorname{scl}}(F, A))=\tilde{\operatorname{scl}}(F, A)$. Then $\tilde{s} c l(F, A) \tilde{\epsilon}$ $\operatorname{SSO}(X)$ and hence by $(1),(F, A) \tilde{\in} \operatorname{S} \beta O(X)$.

(3): Let $(F, A) \tilde{\in} \operatorname{S} \beta O(X)$, so by (1), $\tilde{\operatorname{scl}}(F, A) \tilde{\in} \operatorname{SSO}(X) \tilde{\subseteq} \operatorname{SBO}(X)$. So $\tilde{s} c l(F, A) \tilde{\in} \operatorname{SBO}(X)$. Conversely, let $\tilde{s} c l(F, A) \tilde{\in} \operatorname{SBO}(X) \tilde{\subseteq} S \beta O(X)$, then $\tilde{s} c l(F, A) \tilde{\in} S \beta O(X)$ and thus by $(2),(F, A) \tilde{\in} S \beta O(X)$.

Lemma 3.12. Let $(X, \tau, A)$ be any soft topological space and let $(F, A) \tilde{\in} \operatorname{SS}(X)_{A}$. Then $(F, A) \tilde{\in} \operatorname{SPO}(X)$ if and only if $\tilde{\operatorname{sscl}}(\mathrm{F}, \mathrm{A})=\tilde{\operatorname{sint}}(\tilde{\operatorname{scl}}(\mathrm{F}, \mathrm{A}))$.

Proof. Let $(F, A) \tilde{\in} \operatorname{SPO}(X)$, then $(F, A) \tilde{\subseteq} \tilde{\operatorname{sint}}(\tilde{\operatorname{scl}}(F, A))$ implies that $\tilde{\operatorname{sscl}}(F, A) \tilde{\subseteq} \operatorname{sscl}(\tilde{\operatorname{sint}}(\tilde{\operatorname{scl}}(F, A)))$. Since $\tilde{\operatorname{sint}}(\tilde{\operatorname{scl}}(F, A)) \tilde{\in} \operatorname{SRO}(X)$ and $\operatorname{SRO}(X) \simeq \operatorname{SSC}(X)$ in general, so $\tilde{\operatorname{sint}}(\tilde{\operatorname{scl}}(F, A)) \tilde{\in} \operatorname{SSC}(X)$ and hence

$$
\tilde{\operatorname{sicl}}(\tilde{\operatorname{sint}}(\tilde{\operatorname{scl}}(F, A)))=\tilde{\operatorname{sint}}(\tilde{\operatorname{scl}}(F, A)) .
$$

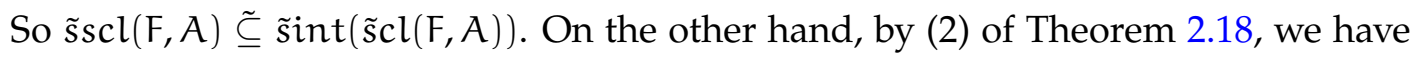

$$
\tilde{\operatorname{sscl}}(F, A)=(F, A) \tilde{\cup} \tilde{\operatorname{sint}}(\tilde{\operatorname{scl}}(F, A)) .
$$

Hence $\tilde{\operatorname{sint}}(\tilde{\operatorname{scl}}(F, A)) \tilde{\subseteq} \tilde{\operatorname{sscl}}(F, A)$. Therefore, $\tilde{\operatorname{sscl}}(F, A)=\tilde{\operatorname{sint}}(\tilde{\operatorname{scl}}(F, A))$.

Conversely, let $\tilde{\operatorname{sscl}}(F, A)=\tilde{\operatorname{sint}}(\tilde{\operatorname{ssl}}(F, A))$, then $\tilde{\operatorname{sscl}}(F, A) \tilde{\subseteq} \tilde{\operatorname{sint}}(\tilde{\operatorname{scl}}(F, A))$ and hence

$$
(F, A) \tilde{\simeq} \tilde{\operatorname{sscl}}(F, A) \tilde{\subseteq} \tilde{\operatorname{sint}}(\tilde{\operatorname{scl}}(F, A)),
$$

which implies that $(F, A) \tilde{\subseteq} \tilde{\operatorname{sint}}(\tilde{\operatorname{scl}}(F, A))$. Therefore, $(F, A) \tilde{\in} \operatorname{SPO}(X)$.

Lemma 3.13. Let $(F, A)$ and $(K, A)$ be any two subsets of a soft topological space $(X, \tau, A)$. Then

$$
\tilde{\operatorname{sint}}(\tilde{\operatorname{scl}}((F, A) \tilde{\cap}(K, A))) \tilde{\subseteq} \tilde{\operatorname{sint}}(\tilde{\operatorname{scl}}(F, A)) \tilde{\cap} \tilde{\operatorname{sint}}(\tilde{\operatorname{scl}}(K, A)) .
$$

Proof. The proof is obvious and hence it is omitted.

Theorem 3.14. Let $(X, \tau, A)$ be a soft topological space. If $(F, A)$ is soft open subset of $X$ and $(K, A)$ is any soft subset of $\mathrm{X}$, then

$$
\tilde{\operatorname{sint}}(\tilde{\operatorname{scl}}(F, A)) \tilde{\cap} \tilde{\operatorname{sint}}(\tilde{\operatorname{scl}}(K, A))=\tilde{\operatorname{sint}}(\tilde{\operatorname{scl}} \operatorname{l}(F, A) \tilde{\cap}(K, A))) \text {. }
$$

Proof. It is enough to prove $\tilde{\operatorname{sint}}(\tilde{\operatorname{s}} \mathrm{cl}(F, A)) \tilde{\cap} \tilde{\operatorname{sint}}(\tilde{\operatorname{scl}}(K, A)) \tilde{\subseteq} \tilde{\operatorname{sint}}(\tilde{\operatorname{scl}}((F, A) \tilde{\cap}(K, A)))$ since the converse is similar to Lemma 3.13. Since $(F, A)$ is soft open subset of a soft space $X$, then by using Lemma 2.12, we have

$$
\begin{aligned}
& \tilde{\operatorname{sint}}(\tilde{\operatorname{scl}}(F, A)) \tilde{\operatorname{sint}} \sin \operatorname{cl}(K, A)) \tilde{\subseteq} \tilde{\operatorname{sint}}[\tilde{\operatorname{scl}} \operatorname{cl}(F, A) \tilde{\operatorname{sint}} \sin \operatorname{sl}(K, A))] \\
& \tilde{\subseteq} \operatorname{sint}(\tilde{\operatorname{scl}}[(F, A) \tilde{\cap} \tilde{\operatorname{sint}}(\tilde{\operatorname{scl}}(K, A))]) \\
& \tilde{\subseteq} \tilde{\operatorname{sint}}(\tilde{\operatorname{scl}}[(F, A) \tilde{\cap} \tilde{s} \operatorname{cl}(K, A)]) \\
& \tilde{\subseteq} \operatorname{sint}(\tilde{\operatorname{scl}}((F, A) \tilde{\cap}(K, A))) \text {. }
\end{aligned}
$$

So $\tilde{\operatorname{sint}}(\tilde{\operatorname{scl}}(F, A)) \tilde{\cap} \tilde{\operatorname{sint}}(\tilde{\operatorname{scl}}(K, A))=\tilde{\operatorname{sint}}(\tilde{\operatorname{scl}}((F, A) \tilde{\cap}(K, A)))$. This completes the proof.

From Theorem 3.14, we have the following corollary.

Corollary 3.15. If $(\mathrm{F}, \mathrm{A})$ and $(\mathrm{K}, \mathrm{A})$ are soft open subsets of a soft topological space $(\mathrm{X}, \tau, \mathrm{A})$, then 


$$
\tilde{\operatorname{sint}}(\tilde{\operatorname{scl}}(F, A)) \tilde{\cap} \tilde{\operatorname{sint}}(\tilde{\operatorname{scl}}(K, A))=\tilde{\operatorname{sint}}(\tilde{\operatorname{scl}}((F, A) \tilde{\cap}(K, A)))
$$

Proof. It is clear.

Corollary 3.16. If $(G, A)$ and $(H, A)$ are soft closed subsets of a soft topological space $(X, \tau, A)$, then

$$
\tilde{\operatorname{s}} \operatorname{cl}(\tilde{\operatorname{sint}}(G, A)) \tilde{U} \tilde{\operatorname{scl}}(\tilde{\operatorname{sint}}(H, A))=\tilde{\operatorname{scl}}(\tilde{\operatorname{sint}}((G, A) \tilde{U}(H, A))) .
$$

Proof. The proof is similar to Corollary 3.15 taking $(F, A)=X \tilde{\backslash}(G, A)$ and $(K, A)=X \tilde{\backslash}(F, A)$.

\section{Soft extremally disconnected spaces}

In this section, we introduce a new class of soft space called soft extremally disconnected, and we obtain several characterizations of soft extremally disconnected spaces by utilizing classes of soft sets.

Definition 4.1. A soft topological space $(X, \tau, A)$ is said to be soft extremally disconnected if the soft closure of every soft open set of $X$ is soft open in $X$, or equivalently, if the soft interior of every soft closed set of $X$ is soft closed in $X$.

In the following theorem, a soft extremally disconnected space $X$ is equivalent to every two disjoint soft open sets of $X$ have disjoint soft closures.

Theorem 4.2. A soft space $X$ is soft extremally disconnected if and only if $\tilde{\operatorname{scl}}(\mathrm{F}, \mathrm{A}) \tilde{\cap} \tilde{\operatorname{scl}}(\mathrm{K}, \mathrm{A})=\tilde{\phi}$ for every soft open subsets $(F, A)$ and $(K, A)$ of $X$ with $(F, A) \tilde{\cap}(K, A)=\tilde{\phi}$.

Proof. Suppose $(F, A)$ and $(K, A)$ are two soft open subsets of a soft extremally disconnected space $X$ such that $(F, A) \tilde{\cap}(K, A)=\tilde{\phi}$. Then by Lemma 3.1, $\tilde{\operatorname{scl}}(F, A) \tilde{\cap}(K, A)=\tilde{\phi}$ which implies that

$$
\tilde{\operatorname{sint}}(\tilde{\operatorname{scl}}(F, A)) \tilde{\cap} \tilde{\operatorname{scl}}(K, A)=\tilde{\phi},
$$

and hence $\tilde{\operatorname{scl}}(\mathrm{F}, \mathrm{A}) \tilde{\cap} \tilde{\operatorname{scl}}(\mathrm{K}, \mathrm{A})=\tilde{\phi}$.

Conversely, let $(O, A)$ be any soft open subset of a soft space $X$, then $X \tilde{\Upsilon}(O, A)$ is soft closed set and hence $\tilde{\operatorname{sint}}(X \tilde{\backslash}(O, A))$ is soft open set such that $(O, A) \tilde{\cap} \operatorname{sint}(X \tilde{\backslash}(O, A))=\tilde{\phi}$. Then by hypothesis, we have $\tilde{s} \operatorname{cl}(O, A) \tilde{\cap} \tilde{\operatorname{scl}}(\tilde{\operatorname{sint}}(X \tilde{\backslash}(O, A)))=\tilde{\phi}$ which implies that $\tilde{\operatorname{scl}}(O, A) \tilde{\cap} \tilde{\operatorname{scl}}(X \tilde{\bar{s}} \tilde{c l}(O, A))=\tilde{\phi}$ and hence $\tilde{\operatorname{scl}}(O, A) \tilde{\cap} X \tilde{\backslash} \tilde{\operatorname{sint}}(\tilde{\operatorname{scl}}(O, A))=\tilde{\phi}$. This means that $\tilde{\operatorname{scl}}(O, A) \tilde{\subseteq} \tilde{\operatorname{sint}}(\tilde{\operatorname{s}} \operatorname{cl}(O, A))$. Since

$$
\tilde{\operatorname{sint}}(\tilde{\operatorname{scl}}(O, A)) \tilde{\subseteq} \operatorname{scl}(O, A),
$$

in general, then $\tilde{\operatorname{s}} \operatorname{cl}(O, A)=\tilde{\operatorname{sint}}(\tilde{\operatorname{s}} \mathrm{cl}(O, A))$. So $\tilde{\operatorname{scl}}(O, A)$ is soft open set in $X$. Therefore, $X$ is soft extremally disconnected space.

Some fundamental characterizations of soft extremally disconnected spaces are given in the following theorems.

Theorem 4.3. Let $(X, \tau, A)$ be a soft topological space. Then the following properties are equivalent:

1. $X$ is soft extremally disconnected.

2. $\tilde{\operatorname{scl}}(F, A) \tilde{\cap} \tilde{\operatorname{scl}}(K, A)=\tilde{\operatorname{scl}}((F, A) \tilde{\cap}(K, A))$ for every soft open subsets $(F, A)$ and $(K, A)$ of $X$.

3. $\tilde{\operatorname{scl}}(\mathrm{F}, \mathrm{A}) \tilde{\cap} \tilde{\operatorname{scl}}(\mathrm{K}, \mathrm{A})=\tilde{\operatorname{s} c l}((\mathrm{~F}, \mathrm{~A}) \tilde{\cap}(\mathrm{K}, \mathrm{A}))$ for every soft regular-open subsets $(\mathrm{F}, \mathrm{A})$ and $(\mathrm{K}, \mathrm{A})$ of $\mathrm{X}$.

4. $\operatorname{sint}(G, A) \tilde{\cup} \tilde{\operatorname{sint}}(H, A)=\tilde{\operatorname{sint}}((G, A) \tilde{\cup}(H, A))$ for every soft regular-closed subsets $(G, A)$ and $(H, A)$ of $X$.

5. $\tilde{\operatorname{sint}}(G, A) \tilde{\cup} \tilde{\operatorname{sint}}(H, A)=\tilde{\operatorname{sint}}((G, A) \tilde{\cup}(H, A))$ for every soft closed subsets $(G, A)$ and $(H, A)$ of $X$. 
Proof.

$(1) \Rightarrow(2)$ : Let $(F, A)$ and $(K, A)$ be any two soft open subsets of a soft extremally disconnected space $X$. Then by Corollary 3.15,

$$
\begin{aligned}
\tilde{\operatorname{scl}}(F, A) \tilde{\cap} \tilde{\operatorname{scl}}(K, A) & =\tilde{\operatorname{sint}}(\tilde{\operatorname{scl}}(F, A)) \tilde{\cap} \tilde{\operatorname{sint}}(\tilde{\operatorname{scl}}(K, A)) \\
& =\tilde{\operatorname{sint}}(\tilde{\operatorname{scl}}((F, A) \cap(K, A)))=\tilde{\operatorname{scl}}((F, A) \tilde{\cap}(K, A)) .
\end{aligned}
$$

$(2) \Rightarrow(3)$ : It is clear since every soft regular-open set is soft open.

$(3) \Leftrightarrow(4)$ : Let $(G, A)$ and $(H, A)$ be two soft regular-closed subsets of $X$. Then $X \tilde{\backslash}(G, A)$ and $X \tilde{\backslash}(H, A)$ are soft regular-open sets. By (3) and Lemma 2.11, we have

$$
\begin{aligned}
& \tilde{\operatorname{scl}}(X \tilde{\backslash}(G, A)) \tilde{\cap} \tilde{s} \operatorname{cl}(X \tilde{\Upsilon}(H, A))=\tilde{\operatorname{scl}}(X \tilde{\backslash}(G, A) \tilde{\cap} X \tilde{\backslash}(H, A))
\end{aligned}
$$

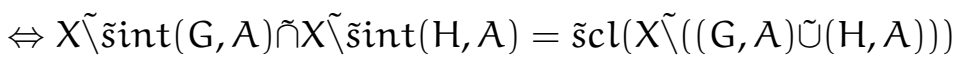

$$
\begin{aligned}
& \Leftrightarrow X \tilde{\Upsilon}(\tilde{\operatorname{sint}}(G, A) \tilde{\cup} \tilde{\operatorname{sint}}(H, A))=X \tilde{\Upsilon} \tilde{\operatorname{sint}}((G, A) \tilde{\cup}(H, A)) \\
& \Leftrightarrow \tilde{\operatorname{sint}}(G, A) \tilde{\cup} \tilde{\operatorname{sint}}(H, A)=\tilde{\operatorname{sint}}((G, A) \tilde{\cup}(H, A)) \text {. }
\end{aligned}
$$

$(4) \Rightarrow(5)$ : Let $(G, A)$ and $(H, A)$ be two soft closed subsets of $X$. Then $\tilde{\operatorname{scl}}(\tilde{\operatorname{sint}}(G, A))$ and $\tilde{\operatorname{scl}}(\tilde{\operatorname{sint}}(H, A))$ are soft regular-closed sets. Then by (4) and Corollary 3.16, we get

$$
\tilde{\operatorname{sint}}(\tilde{\operatorname{scl}}(\tilde{\operatorname{sint}}(G, A))) \tilde{\cup} \tilde{\operatorname{sint}}(\tilde{\operatorname{scl}} \operatorname{sint}(H, A)))=\tilde{\operatorname{sint}}[\tilde{\operatorname{scl}}(\tilde{\operatorname{sint}}(G, A)) \tilde{\operatorname{s}} \operatorname{cl}(\tilde{\operatorname{sint}}(H, A))]
$$

and hence $\tilde{\operatorname{sint}}(\tilde{\operatorname{scl}}(\tilde{\operatorname{sint}}(G, A))) \tilde{\cup} \operatorname{sint}(\tilde{\operatorname{scl}}(\tilde{\operatorname{sint}}(H, A)))=\tilde{\operatorname{sint}}(\tilde{\operatorname{scl}}(\tilde{\operatorname{sint}}((G, A) \tilde{U}(H, A))))$. Since $(G, A)$ and $(H, A)$ are soft closed subsets of $X$, then by (2) of Lemma 3.10, we obtain

$$
\tilde{\operatorname{sint}}(\tilde{\operatorname{scl}}(G, A)) \tilde{U} \operatorname{sint}(\tilde{\operatorname{scl}}(H, A))=\tilde{\operatorname{sint}}(\tilde{\operatorname{scl}}((G, A) \tilde{U}(H, A))) .
$$

This implies that $\tilde{\operatorname{sint}}(G, A) \tilde{\cup} \tilde{\operatorname{sint}}(H, A)=\tilde{\operatorname{sint}}((G, A) \tilde{\cup}(H, A))$.

$(5) \Leftrightarrow(2)$ : The proof is similar to (3) $\Leftrightarrow$ (4).

$(2) \Rightarrow(1)$ : Let $(U, A)$ be any soft open subset of a soft space $X$, then $X \tilde{\backslash}(U, A)$ is soft closed set and hence $\tilde{\operatorname{sint}}(X \tilde{\backslash}(U, A))$ is soft open set. Then by (2), we have

$$
\tilde{\operatorname{scl}}(U, A) \tilde{\cap} \tilde{\operatorname{scl}}(\tilde{\operatorname{sint}}(X \tilde{\backslash}(U, A)))=\tilde{\operatorname{scl}}((U, A) \tilde{\cap} \tilde{\operatorname{sint}}(X \tilde{\backslash}(U, A))),
$$

which implies that $\tilde{\operatorname{scl}}(U, A) \tilde{\cap} \tilde{\operatorname{scl}}(X \tilde{\Upsilon} \tilde{\operatorname{scl}}(U, A))=\tilde{\operatorname{scl}}(\tilde{\phi}) \operatorname{since}(U, A) \tilde{\cap} \tilde{\operatorname{sint}}(X \tilde{\backslash}(U, A))=\tilde{\phi}$. Hence $\tilde{\operatorname{scl}}(U, A) \tilde{\cap} X \tilde{\backslash} \tilde{\operatorname{sint}}(\tilde{\operatorname{scl}}(U, A))=\tilde{\phi}$. This means that $\tilde{\operatorname{scl}}(U, A) \tilde{\tilde{s}} \operatorname{int}(\tilde{\operatorname{s}} \operatorname{cl}(U, A))$. Since

$$
\tilde{\operatorname{sint}}(\tilde{\operatorname{scl}}(U, A)) \tilde{\subseteq} \operatorname{scl}(U, A),
$$

in general, then $\tilde{\operatorname{scl}}(U, A)=\tilde{\operatorname{sint}}(\tilde{\operatorname{scl}}(U, A))$. So $\tilde{\operatorname{scl}}(U, A)$ is soft open set in $X$. Therefore, a soft space $X$ is soft extremally disconnected.

Theorem 4.4. The following statements are equivalent for any soft topological space $(X, \tau, A)$ :

1. $(X, \tau, A)$ is soft extremally disconnected.

2. $\tilde{\operatorname{scl}}(U, A) \tilde{\cap} \tilde{\operatorname{scl}}(V, A)=\tilde{\operatorname{scl}}((\mathrm{U}, A) \tilde{\cap}(V, A))$ for every $(U, A),(V, A) \tilde{\in} \operatorname{SRO}(X)$.

3. $\tilde{\operatorname{scl}}(\mathrm{U}, \mathrm{A}) \tilde{\cap} \tilde{\operatorname{scl}}(\mathrm{V}, \mathrm{A})=\tilde{\operatorname{scl}}((\mathrm{U}, \mathrm{A}) \tilde{\cap}(\mathrm{V}, \mathrm{A}))$ for every $(\mathrm{U}, \mathrm{A}) \tilde{\in} \operatorname{SSO}(X)$ and $(\mathrm{V}, \mathrm{A}) \tilde{\in} \operatorname{S} \beta \mathrm{O}(\mathrm{X})$.

Proof.

$(1) \Leftrightarrow(2)$ : See Theorem 4.3 .

$(1) \Rightarrow(3)$ : Clearly, $\tilde{\operatorname{scl}}((F, A) \tilde{\cap}(B, A)) \tilde{\subseteq} \tilde{s c l}(F, A) \tilde{\cap} \tilde{s c l}(B, A)$ for each $(F, A),(B, A) \tilde{\in} \operatorname{SS}(X)_{A}$. Now, let $(\mathrm{U}, \mathrm{A}) \tilde{\in} \mathrm{SSO}(\mathrm{X})$ and $(\mathrm{V}, \mathrm{A}) \tilde{\in} \mathrm{S} \beta \mathrm{O}(\mathrm{X})$. Since $X$ is soft extremally disconnected, then by (2) of Theorem 4.14, 
$\tilde{\operatorname{scl}}(\mathrm{V}, \mathrm{A}) \tilde{\in} \tau$. Hence by applying Lemma 2.12 and Lemma 3.5, we obtain that

$$
\begin{aligned}
\tilde{\operatorname{scl}}(U, A) \tilde{\cap} \tilde{\operatorname{scl}}(V, A) & =\tilde{\operatorname{scl}}(\tilde{\operatorname{sint}}(\mathrm{U}, A)) \tilde{\cap} \tilde{\operatorname{scl}}(\mathrm{V}, A) \\
& \tilde{\subseteq} \tilde{\operatorname{scl}}(\tilde{\operatorname{sint}}(\mathrm{U}, A) \tilde{\cap} \tilde{\operatorname{scl}}(\mathrm{V}, A)) \\
& \subseteq \tilde{s} \operatorname{cl}((U, A) \tilde{\cap}(\mathrm{V}, A)) .
\end{aligned}
$$

Therefore, $\tilde{\operatorname{scl}}(U, A) \tilde{\cap} \tilde{s} c l(V, A)=\tilde{\operatorname{scl}}((U, A) \tilde{\cap}(V, A))$.

$(3) \Rightarrow(1)$ : Conversely, let $(U, A),(V, A) \tilde{\epsilon} \tau$. Then $(U, A) \tilde{\epsilon} \operatorname{SSO}(X)$ and $(V, A) \tilde{\epsilon} \operatorname{SBO}(X)$ and hence by hypothesis, we have $\tilde{s} c l(U, A) \tilde{\cap} \tilde{s} c l(V, A)=\tilde{\operatorname{scl}}((U, A) \tilde{\cap}(V, A))$. Consequently, by Theorem 4.3, we have $(X, \tau, A)$ is soft extremally disconnected space.

It should be noticed from Theorem 4.4 that

i: $(U, A) \tilde{\epsilon}\{\tau, \operatorname{S} \alpha \mathrm{O}(X)\}$ can be substituted for $(\mathrm{U}, \mathrm{A}) \tilde{\in} \operatorname{SSO}(\mathrm{X})$.

ii: $(V, A) \tilde{\epsilon}\{\tau, \operatorname{S} \alpha O(X), \operatorname{SPO}(X), \operatorname{SSO}(X), \operatorname{SBO}(X)\}$ can be substituted for $(V, A) \tilde{\in} \operatorname{S} \beta O(X)$.

Some relations between types of soft sets via soft extremally disconnected space are shown by the following theorem.

Theorem 4.5. The following statements are equivalent for any soft topological space $(X, \tau, A)$.

1. $X$ is soft extremally disconnected.

2. Every soft regular-closed subset of $X$ is soft open in $X$.

3. Every soft regular-closed subset of $X$ is soft $\alpha$-open in $X$.

4. Every soft regular-closed subset of $X$ is soft preopen in $X$.

5. Every soft semiopen subset of $X$ is soft $\alpha$-open in $X$.

6. Every soft semiclosed subset of $X$ is soft $\alpha$-closed in $X$.

7. Every soft semiclosed subset of $X$ is soft preclosed in $X$.

8. Every soft semiopen subset of $X$ is soft preopen in $X$.

9. Every soft $\beta$-open subset of $X$ is soft preopen in $X$.

10. Every soft $\beta$-closed subset of $X$ is soft preclosed in $X$.

11. Every soft $\mathrm{b}$-closed subset of $\mathrm{X}$ is soft preclosed in $\mathrm{X}$.

12. Every soft b-open subset of $\mathrm{X}$ is soft preopen in $\mathrm{X}$.

13. Every soft regular-open subset of $X$ is soft preclosed in $X$.

14. Every soft regular-open subset of $X$ is soft closed in $X$.

15. Every soft regular-open subset of $\mathrm{X}$ is soft $\alpha$-closed in $\mathrm{X}$.

Proof.

$(1) \Rightarrow(2)$ : Let $(F, A)$ be any soft regular-closed subset of a soft extremally disconnected space $X$. Then $(F, A)=\tilde{\operatorname{scl}}(\tilde{\operatorname{sint}}(F, A))$. Since $(F, A)$ is soft regular-closed set, then it is soft closed and hence $(F, A)=$ $\tilde{\operatorname{scl}}(\tilde{\operatorname{sint}}(F, A))=\tilde{\operatorname{sint}}(F, A)$. Therefore, $(F, A)$ is soft open set in $X$.

The implications (2) $\Rightarrow(3)$ and (3) $\Rightarrow(4)$ are clear since every soft open set is soft $\alpha$-open and every soft $\alpha$-open set is soft preopen.

$(4) \Rightarrow(5)$ : Let $(K, A)$ be a soft semiopen set. So $(K, A) \tilde{\subseteq} \tilde{\operatorname{scl}}(\tilde{\operatorname{sint}}(K, A))$. Since $\tilde{\operatorname{scl}}(\tilde{\operatorname{sint}}(K, A))$ is soft regular-closed set, then by $(4), \tilde{\operatorname{scl}}(\tilde{\operatorname{sint}}(K, A))$ is soft preopen and hence

$$
\tilde{\operatorname{scl}}(\tilde{\operatorname{sint}}(K, A)) \tilde{\subseteq} \tilde{\operatorname{sint}}(\tilde{\operatorname{scl}}(\tilde{\operatorname{scl}}(\tilde{\operatorname{sint}}(K, A))))=\tilde{\operatorname{sint}}(\tilde{\operatorname{scl}}(\tilde{\operatorname{sint}}(K, A))) .
$$

So $(K, A) \tilde{\subseteq} \tilde{\operatorname{sint}}(\tilde{\operatorname{s}} \operatorname{cl}(\tilde{\operatorname{sint}}(K, A)))$. Therefore, $(K, A)$ is soft $\alpha$-open set.

The implications (5) $\Leftrightarrow(6),(6) \Rightarrow(7),(7) \Leftrightarrow(8),(9) \Leftrightarrow(10),(10) \Rightarrow(11),(11) \Leftrightarrow(12)$ and (14) $\Rightarrow$ (15) are obvious. 
$(8) \Rightarrow(9)$ : Let $(G, A)$ be a soft $\beta$-open set. Then by (1) of Theorem $3.11, \tilde{s} c l(G, A)$ is soft semiopen set. So by (8), $\tilde{\operatorname{scl}}(G, A)$ is soft preopen set. So $\tilde{\operatorname{scl}}(G, A) \tilde{\simeq} \tilde{\operatorname{sint}}(\tilde{\operatorname{scl}}(\tilde{\operatorname{scl}}(G, A)))=\tilde{\operatorname{sint}}(\tilde{\operatorname{scl}}(G, A))$ and hence $(G, A) \tilde{\subseteq} \tilde{\operatorname{sint}}(\tilde{\operatorname{scl}}(G, A))$. Therefore, $(G, A)$ is soft preopen set in $X$.

$(12) \Rightarrow(13)$ : Let $(H, A)$ be a soft regular-open set. Then $(H, A)$ is soft $\beta$-open set. By (3) of Theorem 3.11, $\tilde{\operatorname{s}} \mathrm{cl}(H, A)$ is soft b-open set. Thus by $(12), \tilde{\operatorname{s}} \operatorname{cl}(H, A)$ is soft preopen. So

$$
\tilde{\operatorname{scl}}(H, A) \tilde{\subseteq} \tilde{\operatorname{sint}}(\tilde{\operatorname{scl}}(\tilde{\operatorname{scl}}(H, A)))=\tilde{\operatorname{sint}}(\tilde{\operatorname{scl}}(H, A)) .
$$

Since $(H, A)$ is soft regular-open set. Hence $\tilde{s} \operatorname{cl}(H, A) \tilde{\subseteq}(H, A)$. Since $(H, A) \tilde{\subseteq} \tilde{\operatorname{scl}}(H, A)$, then $\tilde{s} c l(H, A)=$ $(H, A)$. This means that $(H, A)$ is soft closed and hence it is soft preclosed.

$(13) \Rightarrow(14)$ : Let $(U, A)$ be a soft regular-open set. Then by (13), $(U, A)$ is soft preclosed set. So $\tilde{\operatorname{scl}}(\tilde{\operatorname{sint}}(U, A)) \tilde{\subseteq}(U, A)$. Since $(U, A)$ is soft regular-open set, then $(U, A)$ is soft open. Hence $\tilde{s} c l(U, A)$ $\tilde{\subseteq}(U, A)$. But in general $(U, A) \tilde{\subseteq} \tilde{\operatorname{scl}}(U, A)$. Therefore, $\tilde{\operatorname{scl}}(U, A)=(U, A)$. This means that $(U, A)$ is soft closed.

$(15) \Rightarrow(1)$ : Let $(V, A)$ be any soft open set of $X$. Then $\tilde{\operatorname{sint}}(\tilde{\operatorname{scl}}(\mathrm{V}, \mathrm{A}))$ is soft regular-open set. By (15), $\tilde{\operatorname{sint}}(\tilde{\operatorname{scl}}(V, A))$ is soft $\alpha$-closed. So

$$
\tilde{\operatorname{scl}}(\tilde{\operatorname{sint}}(\tilde{\operatorname{scl}}(\tilde{\operatorname{sint}}(\tilde{\operatorname{scl}}(\mathrm{V}, \mathrm{A}))))) \tilde{\subseteq} \tilde{\operatorname{sint}}(\tilde{\operatorname{s}} \operatorname{cl}(V, A)) .
$$

By (1) of Lemma 3.10, we get $\tilde{\operatorname{scl}}(\mathrm{V}, \mathrm{A}) \tilde{\subseteq} \operatorname{sint}(\tilde{\operatorname{scl}}(\mathrm{V}, \mathrm{A}))$. But $\tilde{\operatorname{sint}}(\tilde{\operatorname{s}} \mathrm{cl}(\mathrm{V}, \mathrm{A})) \tilde{\subseteq} \operatorname{scl}(\mathrm{V}, \mathrm{A})$ in general. Then $\tilde{\operatorname{scl}}(V, A)=\tilde{\operatorname{sint}}(\tilde{\operatorname{scl}}(V, A))$ and hence $\tilde{\operatorname{scl}}(V, A)$ is soft open set of $X$. Therefore, $X$ is soft extremally disconnected space.

Since the relation between soft regular-open and soft regular-closed sets are independent, but they are equivalent when a soft space $X$ is soft extremally disconnected which is shown in the following remark.

Remark 4.6. A soft space $X$ is soft extremally disconnected if and only if $\operatorname{SRO}(X)=\operatorname{SRC}(X)$.

Theorem 4.7. The following conditions are equivalent for any soft topological space $(X, \tau, A)$.

1. $(X, \tau, A)$ is soft extremally disconnected.

2. The soft closure of every soft $\beta$-open set of $(X, \tau, A)$ is soft regular-open in $(X, \tau, A)$.

3. The soft closure of every soft b-open set of $(X, \tau, A)$ is soft regular-open in $(X, \tau, A)$.

4. The soft closure of every soft preopen set of $(X, \tau, A)$ is soft regular-open in $(X, \tau, A)$.

5. The soft closure of every soft $\alpha$-open set of $(X, \tau, A)$ is soft regular-open in $(X, \tau, A)$.

6. The soft closure of every soft open set of $(X, \tau, A)$ is soft regular-open in $(X, \tau, A)$.

7. The soft closure of every soft regular-open set of $(X, \tau, A)$ is soft regular-open in $(X, \tau, A)$.

Proof.

$(1) \Rightarrow(2)$ : Let $(F, A)$ be a soft $\beta$-open subset of a soft extremally disconnected space $(X, \tau, A)$. Then by (1) and Lemma 3.9, we have $\tilde{\operatorname{scl}}(F, A)=\tilde{\operatorname{scl}}(\tilde{\operatorname{sint}}(\tilde{\operatorname{scl}}(F, A)))=\tilde{\operatorname{sint}}(\tilde{\operatorname{scl}}(F, A)) \operatorname{implies}$ that

$$
\tilde{\operatorname{scl}}(F, A)=\tilde{\operatorname{sint}}(\tilde{\operatorname{scl}}(F, A))=\tilde{\operatorname{sint}}(\tilde{\operatorname{scl}}(\tilde{\operatorname{scl}}(F, A))) .
$$

Hence $\tilde{s} \mathrm{cl}(\mathrm{F}, \mathrm{A})$ is soft regular-open set in $(X, \tau, A)$.

The implications (2) $\Rightarrow(3),(3) \Rightarrow(4),(4) \Rightarrow(5),(5) \Rightarrow(6)$ and $(6) \Rightarrow(7)$ are clear.

$(7) \Rightarrow(1)$ : Let $(O, A)$ be any soft open set of $(X, \tau, A)$, then $\tilde{\operatorname{sint}}(\tilde{\operatorname{s} c l}(O, A))$ is soft regular-open. By (7), $\tilde{\operatorname{scl}}(\tilde{\operatorname{sint}}(\tilde{\operatorname{scl}}(O, A)))$ is soft regular-open set in $(X, \tau, A)$. Since every soft open set is soft $\beta$-open, then $(O, A)$ is soft $\beta$-open and hence by Lemma 3.9, $\tilde{\operatorname{scl}}(O, A)=\tilde{\operatorname{scl}}(\tilde{\operatorname{sint}}(\tilde{\operatorname{scl}}(O, A)))$. This means that $\tilde{\operatorname{scl}}(O, A)$ is soft regular-open set and hence it is soft open. Therefore, $(X, \tau, A)$ is soft extremally disconnected.

Remark 4.8. Soft regular-open set in Theorem 4.7 can be replaced by soft regular-closed set according to Remark 4.6. 
Now, we give further characterizations of soft extremally disconnected spaces by utilizing soft sets.

Theorem 4.9. The following conditions are equivalent for any soft topological space $(X, \tau, A)$.

1. $(X, \tau, A)$ is soft extremally disconnected.

2. The soft closure of every soft semiopen set of $X$ is soft open.

3. The soft $\alpha$-closure of every soft semiopen set of $X$ is soft open.

4. The soft preclosure of every soft semiopen set of $X$ is soft open.

5. The soft closure of every soft semiopen set of $\mathrm{X}$ is soft regular-open.

6. The soft $\alpha$-closure of every soft semiopen set of $X$ is soft regular-open.

7. The soft preclosure of every soft semiopen set of $\mathrm{X}$ is soft regular-open.

Proof.

$(1) \Rightarrow(2)$ : Let $(F, A)$ be any soft semiopen set in a soft extremally disconnected space $(X, \tau, A)$. Then by Lemma 3.5, we have $\tilde{\operatorname{scl}}(F, A)=\tilde{\operatorname{s}} \operatorname{cl}(\tilde{\operatorname{sint}}(F, A))$. Hence $\tilde{\operatorname{scl}}(\tilde{\operatorname{sint}}(F, A))$ is soft open and thus $\tilde{\operatorname{scl}}(F, A)$ is soft open.

$(2) \Rightarrow(1)$ and $(5) \Rightarrow(2)$ : It is clear.

$(2) \Rightarrow(5)$ : Let $(F, A)$ be a soft semiopen set in $(X, \tau, A)$. Then by $(2), \tilde{s} c l(F, A)$ is soft open and hence $\tilde{s} c l(F, A)=\tilde{\operatorname{sint}}(\tilde{\operatorname{scl}}(F, A))$. Therefore, $\tilde{\operatorname{scl}}(F, A)$ is soft regular-open.

$(2) \Leftrightarrow(3) \Leftrightarrow(4)$ and $(5) \Leftrightarrow(6) \Leftrightarrow(7)$ : These follow easily from Corollary 3.7.

Theorem 4.10. Let $(X, \tau, A)$ be any space. Then the following statements are equivalent:

1. $\mathrm{X}$ is soft extremally disconnected.

2. $\tilde{\operatorname{sscl}}(\mathrm{F}, \mathrm{A})=\tilde{\operatorname{scl}}(\mathrm{F}, \mathrm{A})$ for each soft semiopen set $(\mathrm{F}, \mathrm{A})$ in $\mathrm{X}$.

3. The soft semiclosure of every soft semiopen set in $X$ is soft closed.

4. $\tilde{\operatorname{sint}}(\mathrm{F}, \mathrm{A})=\tilde{\operatorname{sint}}(\mathrm{F}, \mathrm{A})$ for each soft semiclosed set $(\mathrm{F}, \mathrm{A})$ in $\mathrm{X}$.

5. The soft semiinterior of every soft semiclosed set in $\mathrm{X}$ is soft open.

Proof.

$(1) \Rightarrow(2)$ : In general, we have $\tilde{\operatorname{sscl}}(S, A) \tilde{\simeq} \tilde{\operatorname{scl}}(S, A)$ for every soft subset $(S, A)$ of $X$. Thus, it is sufficient to show that $\tilde{\operatorname{scl}}(F, A) \tilde{\subseteq} \operatorname{sscl}(F, A)$ for each soft semiopen set $(F, A)$ of $X$. Let $(F, A) \tilde{\in} \operatorname{SSO}(X)$ and $x \tilde{\notin}$ $\tilde{\operatorname{sscl}}(F, A)$, then there exists a soft semiopen set $(U, A)$ containing $x$ such that $(F, A) \tilde{\cap}(U, A)=\tilde{\phi}$ and hence $\tilde{\operatorname{sint}}(F, A) \tilde{\cap} \tilde{\operatorname{sint}}(U, A)=\tilde{\phi}$. Since $X$ is soft extremally disconnected, then we have

$$
\tilde{\operatorname{scl}}(\tilde{\operatorname{sint}}(\mathrm{F}, \mathrm{A})) \tilde{\cap} \tilde{\operatorname{scl}}(\tilde{\operatorname{sint}}(\mathrm{U}, \mathrm{A}))=\tilde{\phi} \text {. }
$$

Since $(F, A)$ is soft semiopen, then $(F, A) \tilde{\cap} \tilde{\operatorname{scl}}(\tilde{\operatorname{sint}}(U, A))=\tilde{\phi}$. Since $\tilde{\sin } \ln (\tilde{\operatorname{sint}}(U, A))$ is soft open set containing $x$. Thus, $x \tilde{\notin} \tilde{\operatorname{scl}}(F, A)$ and hence $\tilde{s} c l(F, A) \tilde{\subseteq} \tilde{\operatorname{sccl}}(F, A)$. Therefore, $\tilde{\operatorname{sscl}}(F, A) \tilde{\subseteq} \tilde{\operatorname{scl}}(F, A)$.

(2) $\Rightarrow(3)$ and (4) $\Rightarrow(5)$ : It is obvious.

$(3) \Rightarrow(1)$ : Let $(F, A)$ be any soft open set in $X$. Then $(F, A)$ is soft semiopen. By (3), $\operatorname{sscl}(F, A)$ is soft

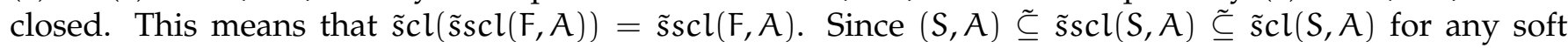
subset $(S, A)$ of $X$, in general. So $\tilde{\operatorname{scl}}(\tilde{\operatorname{sscl}}(S, A))=\tilde{\operatorname{scl}}(S, A)$. Then, we get $\tilde{\operatorname{sscl}}(S, A)=\tilde{\operatorname{s}} \operatorname{cl}(S, A)$. Now, since $(F, A)$ is soft open, then $(F, A)$ is soft preopen. Then by Lemma 3.12, $\tilde{\operatorname{sscl}}(F, A)=\tilde{\operatorname{sint}}(\tilde{\operatorname{scl}}(F, A))$. Therefore, $\tilde{\operatorname{scl}}(F, A)=\tilde{\operatorname{sint}}(\tilde{\operatorname{scl}}(\mathrm{F}, A))$. Consequently, $\tilde{\operatorname{scl}}(F, A)$ is soft open and hence $X$ is soft extremally disconnected.

$(2) \Leftrightarrow(4)$ and $(3) \Leftrightarrow(5)$ : These are clear by using complements.

$(5) \Rightarrow(4)$ : For any soft subset $(S, A)$ of $X$, we have $\tilde{\operatorname{sint}}(S, A) \tilde{\subseteq} \tilde{\operatorname{sint}}(S, A) \tilde{\subseteq}(S, A)$. Then $\tilde{\operatorname{sint}}(\tilde{\operatorname{sint}} \sin (S, A))$ $=\tilde{\operatorname{sint}}(S, A)$. Since $(F, A)$ is soft semiclosed set in $X$, then by $(5), \tilde{s} \operatorname{sint}(F, A)$ is soft open. Therefore, $\tilde{\operatorname{sint}}(\mathrm{F}, A)=\tilde{\operatorname{sint}}(\mathrm{F}, \mathrm{A})$. 
Theorem 4.11. Let $(X, \tau, A)$ be any space. Then the following statements are equivalent:

1. $X$ is soft extremally disconnected.

2. The soft semiclosure of every soft semiopen set in $X$ is soft open.

3. The soft $\mathrm{b}$-closure of every soft semiopen set in $\mathrm{X}$ is soft open.

4. The soft $\mathrm{b}$-closure of every soft semiopen set in $\mathrm{X}$ is soft closed.

5 . The soft $\beta$-closure of every soft semiopen set in $X$ is soft open.

6. The soft $\beta$-closure of every soft semiopen set in $X$ is soft closed.

Proof.

$(1) \Leftrightarrow(2)$ : This is an immediate consequence of (1) and (2) of Theorem 4.9 and (1) and (2) of Theorem 4.10.

(2) $\Leftrightarrow(3) \Leftrightarrow(5)$ : These follow from (1) of Lemma 3.8.

(1) $\Leftrightarrow(4) \Leftrightarrow(6)$ : These follow directly from (1) and (3) of Theorem 4.10, and (1) of Lemma 3.8.

Theorem 4.12. Let $(X, \tau, A)$ be any space. Then the following statements are equivalent:

1. $X$ is soft extremally disconnected.

2. $\tilde{\operatorname{sscl}}(\tilde{\sin } \operatorname{sint}(F, A)) \tilde{\epsilon} \tau$ for every $(F, A) \tilde{\in} \operatorname{SS}(X)_{A}$.

3. $\tilde{\operatorname{sbcl}}(\tilde{\sin } \operatorname{sint}(F, A)) \tilde{\epsilon} \tau$ for every $(F, A) \tilde{\in} \operatorname{SS}(X)_{A}$.

4. $\tilde{s} \beta \operatorname{cl}(\tilde{\sin } \operatorname{sint}(F, A)) \tilde{\epsilon} \tau$ for every $(F, A) \tilde{\in} \operatorname{SS}(X)_{A}$.

Proof. This is an immediate consequence of Theorem 4.11.

The proof of the following theorem follows directly from (1) and (2) of Theorem 4.10, Corollary 3.7 and (1) of Lemma 3.8.

Theorem 4.13. The following statements are equivalent for any soft topological space $(X, \tau, A)$.

1. $X$ is soft extremally disconnected.

2. $\tilde{\operatorname{sscl}}(F, A)=\tilde{\operatorname{sicl}}(F, A)$ for each $(F, A) \tilde{\in} \operatorname{SSO}(X)$.

3. $\operatorname{sscl}(F, A)=\tilde{\operatorname{spcl}}(F, A)$ for each $(F, A) \tilde{\in} \operatorname{SSO}(X)$.

4. $\tilde{s b c l}(F, A)=\tilde{\operatorname{scl}}(F, A)$ for each $(F, A) \tilde{\in} \operatorname{SSO}(X)$.

5. $\tilde{\operatorname{sbcl}}(F, A)=\tilde{\operatorname{s}} \alpha \operatorname{cl}(F, A)$ for each $(F, A) \tilde{\in} \operatorname{SSO}(X)$.

6. $\tilde{\operatorname{sbcl}}(\mathrm{F}, \mathrm{A})=\tilde{\operatorname{spcl}}(\mathrm{F}, \mathrm{A})$ for each $(\mathrm{F}, \mathrm{A}) \tilde{\in} \operatorname{SSO}(X)$.

7. $\tilde{s} \beta \operatorname{cl}(F, A)=\tilde{\operatorname{scl}}(F, A)$ for each $(F, A) \tilde{\in} \operatorname{SSO}(X)$.

8. $\tilde{s} \beta \operatorname{cl}(F, A)=\tilde{\operatorname{s}} \alpha \operatorname{cl}(F, A)$ for each $(F, A) \tilde{\in} \operatorname{SSO}(X)$.

9. $\tilde{s} \beta \operatorname{cl}(F, A)=\tilde{\operatorname{spcl}}(F, A)$ for each $(F, A) \tilde{\in} \operatorname{SSO}(X)$.

Theorem 4.14. The following statements are equivalent for any soft topological space $(X, \tau, A)$.

1. $X$ is soft extremally disconnected.

2. The soft closure of every soft $\beta$-open set of $X$ is soft open.

3. The soft closure of every soft preopen set of $X$ is soft open.

4. The soft $\alpha$-closure of every soft preopen set of $X$ is soft open.

5 . The soft $\alpha$-closure of every soft $\beta$-open set of $X$ is soft open.

Proof.

$(1) \Rightarrow(2)$ : Let $(F, A) \tilde{\in} S \beta O(X)$, then $\tilde{\operatorname{sint}}(\tilde{\operatorname{scl}}(F, A)) \tilde{\in} \tau$. Since $X$ is soft extremally disconnected space, then $\tilde{\operatorname{scl}}(\tilde{\operatorname{sint}}(\tilde{\operatorname{scl}}(F, A))) \tilde{\epsilon} \tau$. By Lemma 3.9, we have $\tilde{\operatorname{scl}}(F, A) \tilde{\in} \tau$.

$(2) \Rightarrow(3)$ : It is clear since every soft preopen set is soft $\beta$-open.

$(3) \Rightarrow(1)$ : It is clear since every soft open set is soft preopen. 
$(2) \Leftrightarrow(5)$ : It follows directly from Lemma 3.3.

(3) $\Leftrightarrow$ (4): It follows from Lemma 3.4.

Lemma 4.15. Let $(F, A)$ be any soft subset of a soft topological space $(X, \tau, A)$. Then the following statements hold:

1. $\tilde{\operatorname{sbcl}}(\tilde{\operatorname{sint}}(F, A))=\tilde{\operatorname{sint}}(\tilde{\operatorname{s}} \operatorname{bcl}(F, A))=\tilde{\operatorname{sint}}(\tilde{\operatorname{scl}}(\tilde{\operatorname{sint}}(F, A)))$.

2. $\tilde{\operatorname{sbint}}(\tilde{\operatorname{scl}}(F, A))=\tilde{\operatorname{scl}}(\tilde{\operatorname{sbint}}(F, A))=\tilde{\operatorname{scl}}(\tilde{\operatorname{sint}}(\tilde{\operatorname{scl}}(F, A)))$.

Proof. (1): By (1), (2) and (3) of Theorem 2.18, we obtain

$$
\begin{aligned}
\tilde{\operatorname{s}} \operatorname{ccl}(\tilde{\operatorname{sint}}(F, A)) & =\tilde{\operatorname{spcl}}(\tilde{\operatorname{sint}}(F, A)) \tilde{\cap} \tilde{\operatorname{scl}}(\tilde{\operatorname{sint}}(F, A)) \\
& =[\tilde{\operatorname{sint}}(F, A) \tilde{\cup} \tilde{\operatorname{s}} \operatorname{cl}(\tilde{\operatorname{sint}}(\tilde{\operatorname{sint}}(F, A)))] \tilde{n}[\tilde{\operatorname{sint}}(F, A) \tilde{\cup} \tilde{\operatorname{sint}}(\tilde{\operatorname{s}} \operatorname{cl}(\tilde{\operatorname{sint}}(F, A)))] \\
& =\tilde{\operatorname{scl}}(\tilde{\operatorname{sint}}(F, A)) \tilde{\operatorname{sint}}(\tilde{\operatorname{scl}}(\tilde{\operatorname{sint}}(F, A))))=\tilde{\operatorname{sint}}(\tilde{\operatorname{scl}}(\tilde{\operatorname{sint}}(F, A))) .
\end{aligned}
$$

Again, by (3) of Theorem 2.18, we have

$$
\begin{aligned}
\tilde{\operatorname{sint}}(\tilde{\operatorname{sbcl}}(F, A)) & =\tilde{\operatorname{sint}}(\tilde{\operatorname{spcl}}(F, A) \tilde{\cap} \tilde{s} \operatorname{scl}(F, A)) \\
& =\tilde{\operatorname{sint}}(\tilde{\operatorname{spcl}}(F, A)) \tilde{\cap} \tilde{\operatorname{sint}}(\tilde{\operatorname{sscl}}(F, A)) \\
& =\tilde{\operatorname{sint}}(\tilde{\operatorname{scl}}(\tilde{\operatorname{sint}}(F, A))) \tilde{\operatorname{sint}}(\tilde{\operatorname{scl}}(F, A)) \\
& =\tilde{\operatorname{sint}}(\tilde{\operatorname{s}} \operatorname{cl}(\tilde{\operatorname{sint}}(F, A))) .
\end{aligned}
$$

(2): It follows from (1).

Theorem 4.16. Let $(X, \tau, A)$ be any soft space. Then the following statements are equivalent:

1. $X$ is soft extremally disconnected.

2. $\tilde{\operatorname{scl}}(\tilde{\operatorname{sbint}}(\mathrm{F}, A)) \tilde{\epsilon} \tau$ for every $(F, A) \tilde{\in} \mathrm{S} \beta \mathrm{O}(\mathrm{X})$.

3. $\tilde{\operatorname{sbint}}(\tilde{\operatorname{scl}}(\mathrm{F}, A)) \tilde{\in} \tau$ for every $(F, A) \tilde{\in} \mathrm{S} \beta \mathrm{O}(X)$.

4. $\tilde{\operatorname{sint}}(\tilde{\operatorname{sibcl}}(K, A)) \tilde{\in} \mathrm{SC}(X)$ for every $(K, A) \tilde{\in} S \beta C(X)$.

5. $\tilde{\operatorname{sbcl}}(\tilde{\operatorname{sint}}(K, A)) \tilde{\in} \mathrm{SC}(X)$ for every $(K, A) \tilde{\in} S \beta C(X)$.

Proof.

$(1) \Rightarrow(2)$ : Let $(F, A) \tilde{\in} S \beta O(X)$. Then by Lemma 3.9 and (2) of Lemma 4.15, we have

$$
\tilde{\operatorname{scl}}(F, A)=\tilde{\operatorname{scl}}(\tilde{\operatorname{sint}}(\tilde{\operatorname{scl}}(F, A)))=\tilde{\operatorname{scl}}(\tilde{\operatorname{s}} \operatorname{bint}(F, A)) .
$$

Since the soft space $X$ is soft extremally disconnected, then $\tilde{\operatorname{scl}}(F, A) \tilde{\in} \tau$ and hence $\tilde{\operatorname{s} c l}(\tilde{\operatorname{sbint}}(F, A)) \tilde{\in} \tau$.

$(2) \Rightarrow(1)$ : This follows directly from Lemma 3.9, (2) of Lemma 4.15 and (2) of Theorem 4.14.

(2) $\Leftrightarrow(3)$ and (4) $\Leftrightarrow(5)$ : These follow easily from Lemma 4.15.

$(2) \Leftrightarrow(4)$ : It is obvious.

Lemma 4.17. Let $(\mathrm{X}, \tau, \mathrm{A})$ be a soft topological space. Then the following statements are equivalent:

1. $X$ is soft extremally disconnected.

2. $\tilde{\operatorname{scl}}(F, A) \tilde{\cap} \tilde{\operatorname{scl}}(K, A)=\tilde{\phi}$ for every soft open subsets $(F, A)$ and $(K, A)$ of $X$ with $(F, A) \tilde{\cap}(K, A)=\tilde{\phi}$.

3. $\tilde{\operatorname{scl}}(\mathrm{F}, \mathrm{A}) \tilde{\cap} \tilde{\operatorname{scl}}(\mathrm{K}, \mathrm{A})=\tilde{\phi}$ for every soft regular-open subsets $(\mathrm{F}, \mathrm{A})$ and $(\mathrm{K}, \mathrm{A})$ of $\mathrm{X}$ with $(\mathrm{F}, \mathrm{A}) \tilde{\cap}(\mathrm{K}, \mathrm{A})=$ $\tilde{\phi}$.

Proof.

(1) $\Leftrightarrow(2)$ : See Theorem 4.2.

$(2) \Rightarrow(3)$ : Since every soft regular-open set is soft open, then the proof is clear. 
$(3) \Rightarrow(2)$ : Let $(F, A)$ and $(K, A)$ be any two soft open subsets of a soft space $X$ such that $(F, A) \tilde{\cap}(K, A)=\tilde{\phi}$. Then by Lemma 3.1, $(F, A) \tilde{\cap} \tilde{\operatorname{scl}}(K, A)=\tilde{\phi} \operatorname{implies}$ that $\tilde{\operatorname{scl}}(F, A) \tilde{\cap} \tilde{\operatorname{sint}}(\tilde{\operatorname{scl}}(K, A))=\tilde{\phi}$ and hence $\tilde{\operatorname{sint}}(\tilde{\operatorname{scl}}(F, A)) \tilde{\cap} \tilde{\operatorname{sint}}(\tilde{\operatorname{scl}}(K, A))=\tilde{\phi}$. Since $\tilde{\operatorname{sint}}(\tilde{\operatorname{s}} \operatorname{cl}(F, A))$ and $\tilde{\operatorname{sint}}(\tilde{\operatorname{scl}}(K, A))$ are two soft regular-open sets. Then by (3), we obtain $\tilde{\operatorname{scl}}(\tilde{\operatorname{sint}}(\tilde{\operatorname{scl}}(F, A))) \tilde{\cap} \tilde{\operatorname{s}} \operatorname{cl}(\tilde{\operatorname{sint}}(\tilde{\operatorname{scl}}(K, A)))=\tilde{\phi}$. Since $\tilde{\operatorname{sint}}(\tilde{\operatorname{scl}}(F, A))$ and $\tilde{\operatorname{sint}}(\tilde{\operatorname{scl}}(K, A))$ are two soft regular-open sets, then $\tilde{\operatorname{sint}}(\tilde{\operatorname{scl}}(F, A))$ and $\tilde{\operatorname{sint}}(\tilde{\operatorname{scl}}(K, A))$ are two soft $\beta$ open sets. So by Lemma 3.9, we get $\tilde{\operatorname{scl}}(F, A) \tilde{\cap} \tilde{\operatorname{scl}}(K, A)=\tilde{\phi}$. This completes the proof.

Corollary 4.18. A soft space $X$ is soft extremally disconnected if and only if $\tilde{\operatorname{scl}}(F, A) \tilde{\cap} \tilde{\operatorname{scl}}(\tilde{\operatorname{sint}}(\tilde{\operatorname{scl}}(\mathrm{K}, \mathrm{A})))=\tilde{\phi}$ for every soft open subset $(F, A)$ and every soft subset $(K, A)$ of $X$ with $(F, A) \tilde{\cap}(K, A)=\tilde{\phi}$.

Proof. See Lemma 4.17 , since $(F, A)$ and $\tilde{\operatorname{sint}}(\tilde{\operatorname{scl}}(\mathrm{K}, \mathrm{A}))$ are two soft open subsets of $X$ such that $(F, A) \tilde{\cap} \tilde{\operatorname{sint}}(\tilde{\operatorname{scl}}(K, A))=\tilde{\phi}$.

Theorem 4.19. Let $(X, \tau, A)$ be a soft topological space. Then $X$ is soft extremally disconnected if and only if $\tilde{\operatorname{sint}}(\tilde{\operatorname{scl}}(F, A)) \tilde{\cup} \tilde{\operatorname{sint}}(\tilde{\operatorname{scl}}(K, A))=\tilde{\operatorname{sint}}(\tilde{\operatorname{scl}}((F, A) \tilde{\cup}(K, A)))$ for every soft open subsets $(F, A)$ and $(K, A)$ of $\mathrm{X}$.

Proof. Let $(X, \tau, A)$ be a soft extremally disconnected space and let $(F, A)$ and $(K, A)$ be any two soft open subsets of $X$. Then $\tilde{s} c l(F, A)$ and $\tilde{s} c l(K, A)$ are soft closed subsets of $X$. So by (5) of Theorem 4.3, we have $\tilde{\operatorname{sint}}(\tilde{\operatorname{scl}}(F, A)) \tilde{\cup} \tilde{\operatorname{sint}}(\tilde{\operatorname{scl}}(K, A))=\tilde{\operatorname{sint}}(\tilde{\operatorname{scl}}(F, A) \tilde{\cup} \tilde{\operatorname{scl}}(K, A))=\tilde{\operatorname{sint}}(\tilde{\operatorname{s}} \operatorname{cl}((F, A) \tilde{\cup}(K, A)))$.

Conversely, let $(G, A)$ and $(H, A)$ be two soft closed subsets of $X$. Then $\tilde{\operatorname{sint}}(G, A)$ and $\tilde{\operatorname{sint}}(H, A)$ are soft open subsets of $X$. So by hypothesis,

$$
\begin{aligned}
\tilde{\operatorname{sint}}(\tilde{\operatorname{scl}}(\tilde{\operatorname{sint}}(G, A))) \tilde{\cup} \tilde{\operatorname{sint}}(\tilde{\operatorname{scl}}(\tilde{\operatorname{sint}}(H, A))) & =\tilde{\operatorname{sint}}(\tilde{\operatorname{scl}}[\tilde{\operatorname{sint}}(G, A) \tilde{\cup} \tilde{\operatorname{sint}}(H, A)]) \\
& =\tilde{\operatorname{sint}}[\tilde{\operatorname{s}} \operatorname{cl}(\tilde{\operatorname{sint}}(G, A)) \cup \tilde{\operatorname{scl}}(\tilde{\operatorname{sint}}(H, A))] .
\end{aligned}
$$

Since $(G, A)$ and $(H, A)$ are soft closed subsets of $X$, then by Corollary 3.16,

$$
\tilde{\operatorname{sint}}(\tilde{\operatorname{scl}}(\tilde{\operatorname{sint}}(G, A))) \tilde{\cup} \tilde{\operatorname{sint}}(\tilde{\operatorname{scl}}(\tilde{\operatorname{sint}}(H, A)))=\tilde{\operatorname{sint}}(\tilde{\operatorname{scl}}(\tilde{\operatorname{sint}}((G, A) \tilde{U}(H, A)))) .
$$

Hence by (2) of Lemma 3.10, we have

$$
\tilde{\operatorname{sint}}(\tilde{\operatorname{scl}}(G, A)) \tilde{U} \operatorname{sint}(\tilde{\operatorname{scl}}(H, A))=\tilde{\operatorname{sint}}(\tilde{\operatorname{scl}}((G, A) \tilde{U}(H, A))) .
$$

This $\operatorname{implies}$ that $\tilde{\operatorname{sint}}(G, A) \tilde{\cup} \tilde{\operatorname{sint}}(H, A)=\tilde{\operatorname{sint}}((G, A) \tilde{\cup}(H, A))$. Therefore, by (5) of Theorem $4.3, X$ is soft extremally disconnected space.

Theorem 4.20. Let $(X, \tau, A)$ be a soft topological space. Then $X$ is soft extremally disconnected if and only if $\tilde{\operatorname{scl}}(\tilde{\operatorname{sint}}(G, A)) \tilde{\cap} \tilde{\operatorname{scl}}(\tilde{\operatorname{sint}}(H, A))=\tilde{\operatorname{scl}}(\tilde{\operatorname{sint}}((G, A) \tilde{\cap}(H, A)))$ for every soft closed subsets $(G, A)$ and $(H, A)$ of X.

Proof. The proof is similar to Theorem 4.19 by taking $(F, A)=(G, A)^{c}$ and $(K, A)=(H, A)^{c}$.

Theorem 4.21. Let $(X, \tau, A)$ be any space. Then the following are equivalent:

1. $(X, \tau, A)$ is soft extremally disconnected.

2. $\tilde{\operatorname{scl}}(\mathrm{F}, \mathrm{A})=\tilde{\operatorname{s}} \mathrm{cl}(\mathrm{F}, \mathrm{A})$ for every soft $\beta$-open set $(F, A)$ in $X$.

3. $\tilde{\operatorname{sscl}}(\mathrm{F}, \mathrm{A})=\tilde{\operatorname{s}} \alpha \mathrm{cl}(\mathrm{F}, \mathrm{A})$ for each soft $\beta$-open set $(F, A)$ in $X$.

Proof.

$(1) \Rightarrow(2):$ In general $\tilde{\operatorname{sint}}(\tilde{\operatorname{s}} \mathrm{cl}(S, A)) \tilde{\subseteq} \operatorname{sscl}(S, A) \tilde{\subseteq} \tilde{\operatorname{scl}}(S, A)$ for any soft subset $(S, A)$ of $X$. Let $X$ be a soft extremally disconnected and $(F, A)$ be a soft $\beta$-open set of $X$, then by (2) of Theorem $4.14, \tilde{s} c l(F, A)$ is soft open in $X$. This means that $\tilde{s} \operatorname{scl}(F, A)=\tilde{s} \operatorname{cl}(F, A)$ for every soft $\beta$-open set $(F, A)$ of $X$.

$(2) \Rightarrow(1)$ : Let $(U, A)$ and $(V, A)$ be soft open subsets of $X$ such that $(U, A) \tilde{\cap}(V, A)=\tilde{\phi}$. Then we have $\tilde{\operatorname{sscl}}(U, A) \tilde{\cap}(V, A)=\tilde{\phi}$. Since $\tilde{\operatorname{sscl}}(U, A)$ is soft semiopen set in $X$, $\operatorname{so} \tilde{\operatorname{sscl}}(U, A) \tilde{\cap} \tilde{\operatorname{sscl}}(V, A)=\tilde{\phi}$. By using the part (3), we have $\tilde{\operatorname{scl}}(\mathrm{U}, A) \tilde{\cap} \tilde{\operatorname{scl}}(\mathrm{V}, A)=\tilde{\phi}$. Therefore, by Lemma 4.17, $(X, \tau, A)$ is soft 
extremally disconnected.

(2) $\Leftrightarrow(3)$ : These follow easily from Lemma 3.3.

Theorem 4.22. Let $(X, \tau, A)$ be a soft topological space. Then the following statements are equivalent:

1. $X$ is soft extremally disconnected.

2. If $(U, A) \tilde{\in} \operatorname{SSO}(X)$ and $(V, A) \tilde{\in} \operatorname{S\beta O}(X)$ such that $(U, A) \tilde{\cap}(V, A)=\tilde{\phi}$, then $\tilde{\operatorname{scl}}(U, A) \tilde{\cap} \tilde{s} c l(V, A)=\tilde{\phi}$.

3. If $(\mathrm{U}, \mathrm{A}) \tilde{\in} \operatorname{SSO}(\mathrm{X})$ and $(\mathrm{V}, \mathrm{A}) \tilde{\in} \operatorname{SBO}(\mathrm{X})$ such that $(\mathrm{U}, \mathrm{A}) \tilde{\cap}(\mathrm{V}, \mathrm{A})=\tilde{\phi}$, then $\tilde{\operatorname{scl}}(\mathrm{U}, \mathrm{A}) \tilde{\cap} \tilde{\operatorname{scl}}(\mathrm{V}, \mathrm{A})=\tilde{\phi}$.

4. If $(\mathrm{U}, \mathrm{A}) \tilde{\in} \operatorname{SSO}(\mathrm{X})$ and $(\mathrm{V}, \mathrm{A}) \tilde{\in} \operatorname{SSO}(\mathrm{X})$ such that $(\mathrm{U}, \mathrm{A}) \tilde{\cap}(\mathrm{V}, \mathrm{A})=\tilde{\phi}$, then $\tilde{\operatorname{scl}}(\mathrm{U}, \mathrm{A}) \tilde{\cap} \tilde{\mathrm{s}} \mathrm{cl}(\mathrm{V}, \mathrm{A})=\tilde{\phi}$.

5. If $(\mathrm{U}, \mathrm{A}) \tilde{\in} \operatorname{SSO}(\mathrm{X})$ and $(\mathrm{V}, \mathrm{A}) \tilde{\in} \operatorname{SPO}(\mathrm{X})$ such that $(\mathrm{U}, \mathrm{A}) \tilde{\cap}(\mathrm{V}, \mathrm{A})=\tilde{\phi}$, then $\tilde{\operatorname{scl}}(\mathrm{U}, \mathrm{A}) \tilde{\cap} \tilde{\mathrm{s}} \mathrm{cl}(\mathrm{V}, \mathrm{A})=\tilde{\phi}$.

6. If $(\mathrm{U}, \mathrm{A}) \tilde{\in} \operatorname{SSO}(\mathrm{X})$ and $(\mathrm{V}, \mathrm{A}) \tilde{\in} \mathrm{S} \alpha \mathrm{O}(\mathrm{X})$ such that $(\mathrm{U}, \mathrm{A}) \tilde{\cap}(\mathrm{V}, \mathrm{A})=\tilde{\phi}$, then $\tilde{\operatorname{scl}}(\mathrm{U}, \mathrm{A}) \tilde{\cap} \tilde{\operatorname{scl}}(\mathrm{V}, \mathrm{A})=\tilde{\phi}$.

7. If $(U, A) \tilde{\in} \operatorname{SSO}(X)$ and $(V, A) \tilde{\in} \tau$ such that $(U, A) \tilde{\cap}(V, A)=\tilde{\phi}$, then $\tilde{s} \operatorname{cl}(U, A) \tilde{\cap} \tilde{s} \operatorname{cl}(V, A)=\tilde{\phi}$.

8. If $(\mathrm{U}, \mathrm{A}) \tilde{\in} \operatorname{SSO}(\mathrm{X})$ and $(\mathrm{V}, \mathrm{A}) \tilde{\in} \operatorname{SRO}(\mathrm{X})$ such that $(\mathrm{U}, \mathrm{A}) \tilde{\cap}(\mathrm{V}, \mathrm{A})=\tilde{\phi}$, then $\tilde{\operatorname{scl}}(\mathrm{U}, \mathrm{A}) \tilde{\cap} \tilde{\operatorname{scl}}(\mathrm{V}, \mathrm{A})=\tilde{\phi}$.

Proof.

$(1) \Rightarrow(2)$ : Suppose that $(U, A) \tilde{\epsilon} \operatorname{SSO}(X)$ and $(V, A) \tilde{\epsilon} S \beta O(X)$ such that $(U, A) \tilde{\cap}(V, A)=\tilde{\phi}$. Then $\tilde{\operatorname{sint}}(U, A) \tilde{\cap} \tilde{s} c l(V, A)=\tilde{\phi}$. By (1) and (2) of Theorem 4.14, $\tilde{\operatorname{scl}}(V, A)$ is soft open in $X$. Hence $\tilde{\operatorname{scl}}(\tilde{\operatorname{sint}}(\mathrm{U}, A))$ $\tilde{\cap} \tilde{\operatorname{scl}}(\mathrm{V}, \mathrm{A})=\tilde{\phi}$. Since $(\mathrm{U}, \mathrm{A}) \tilde{\in} \operatorname{SSO}(\mathrm{X})$, then by Lemma 3.5, we obtain that

$$
\tilde{\operatorname{scl}}(\mathrm{U}, \mathrm{A}) \tilde{\cap} \tilde{\operatorname{scl}}(\mathrm{V}, \mathrm{A})=\tilde{\phi} .
$$

The implications (2) $\Rightarrow(3),(3) \Rightarrow(4),(4) \Rightarrow(6),(3) \Rightarrow(5),(5) \Rightarrow(6),(6) \Rightarrow(7)$ and (7) $\Rightarrow(8)$ are obvious. $(4) \Rightarrow(5):$ Let $(U, A) \tilde{\in} \operatorname{SSO}(X)$ and $(V, A) \tilde{\in} \operatorname{SPO}(X)$ such that $(U, A) \tilde{\cap}(V, A)=\tilde{\phi}$. Then

$$
\tilde{\operatorname{scl}}(V, A)=\tilde{\operatorname{scl}}(\tilde{\operatorname{sint}}(\tilde{\operatorname{scl}}(V, A))),
$$

and

$$
\tilde{\operatorname{sint}}(\tilde{\operatorname{scl}}(\mathrm{V}, \mathrm{A})) \tilde{\in} \operatorname{SSO}(X) .
$$

Thus, by (4), we have

$$
\tilde{\operatorname{s}} \operatorname{cl}(U, A) \tilde{\cap} \tilde{\operatorname{scl}}(\tilde{\operatorname{sint}}(\tilde{\operatorname{scl}} \operatorname{l}(\mathrm{V}, \mathrm{A})))=\tilde{\phi} .
$$

This implies that $\tilde{s} c l(U, A) \tilde{\cap} \tilde{s} c l(V, A)=\tilde{\phi}$.

$(8) \Rightarrow(1)$ : Let $(U, A),(V, A) \tilde{\in} \operatorname{SRO}(X)$ such that $(U, A) \tilde{\cap}(V, A)=\tilde{\phi}$. Then $(U, A) \tilde{\in} \operatorname{SSO}(X)$ and hence by (10), $\tilde{\operatorname{scl}}(\mathrm{U}, A) \tilde{\cap} \tilde{\operatorname{scl}}(\mathrm{V}, A)=\tilde{\phi}$. Therefore, by Lemma 4.17, $\mathrm{X}$ is soft extremally disconnected.

It should be noticed from Theorem 4.22 that

i. $(U, A),(V, A) \tilde{\in}\{\operatorname{S} \alpha O(X), \tau, \operatorname{SRO}(X)\}$ can be substituted for $(U, A),(V, A) \tilde{\in} \operatorname{SSO}(X)$.

ii. The notions $\tilde{s} \alpha c l$ can be substituted for $\tilde{s} c l$.

iii. $\tilde{\operatorname{spcl}}(S, A)$ can be substituted for $\tilde{\operatorname{scl}}(S, A)$ for every $(S, A) \tilde{\in} \operatorname{SSO}(X)$.

\section{References}

[1] M. Akdag, A. Ozkan, Soft $\alpha$-open sets and soft $\alpha$-continuous functions, Abstr. Appl. Anal., 2014 (2014), 7 pages. 2, $2.14,2.16,2.17$

[2] M. Akdag, A. Ozkan, Soft b-open sets and soft b-continuous functions, Math. Sci. (Springer), 8 (2014), 9 pages. 5, 2.14, $2.16,2.17,3$

[3] I. Arockiarani, A. A. Lancy, Generalized soft $g \beta$-closed sets and soft gs $\beta$-closed sets in soft topological spaces, Int. J. Math. Arch., 4 (2013), 17-23. 6, 2.14, 2.16, 2.17, 4

[4] B. Chen, Soft semi-open sets and related properties in soft topological spaces, Appl. Math. Inf. Sci., 7 (2013), 287-294. 3, $2.14,2.16,2.17,2$ 
[5] D.-G. Chen, E. C. C. Tsang, D. S. Yeung, X.-Z. Wang, The parameterization reduction of soft sets and its applications, Comput. Math. Appl., 49 (2005), 757-763. 1

[6] S. Das, S. K. Samanta, Soft metric, Ann. Fuzzy Math. Inform., 6 (2013), 77-94. 2.8

[7] F. Feng, Y. B. Jun, X.-Z. Zhao, Soft semirings, Comput. Math. Appl., 56 (2008), 2621-2628. 2.6

[8] D. N. Georgiou, A. C. Megaritis, V. I. Petropoulos, On soft topological spaces, Appl. Math. Inf. Sci., 7 (2013), 18891901. 1

[9] S. Hussain, B. Ahmad, Some properties of soft topological spaces, Comput. Math. Appl., 62 (2011), 4058-4067. 2, 2.11

[10] G. Ilango, M. Ravindran, On soft preopen sets in soft topological spaces, Int. J. Math. Res., 5 (2013), 399-409. 4, 2.14, 2.16, 2.17, 1

[11] A. Kandil, O. A. E. Tantawy, S. A. El-Sheikh, A. M. Abd El-latif, $\gamma$-operation and decompositions of some forms of soft continuity in soft topological spaces, Ann. Fuzzy Math. Inform., 7 (2014), 181-196. 3.5

[12] A. Kandil, O. A. E. Tantawy, S. A. El-Sheikh, A. M. Abd El-latif, Soft semi compactness via soft ideals, Appl. Math. Inf. Sci., 8 (2014), 2297-2306. 1

[13] Z. Kong, L.-Q. Gao, L.-F. Wong, S. Li, The normal parameter reduction of soft sets and its algorithm, Comput. Math. Appl., 56 (2008), 3029-3037. 1

[14] P. K. Maji, R. Biswas, A. R. Roy, Soft set theory, Comput. Math. Appl., 45 (2003), 555-562. 1, 2.2, 2.3, $2.4,2.5$

[15] P. K. Maji, A. R. Roy, R. Biswas, An application of soft sets in a decision making problem, Comput. Math. Appl., 44 (2002), 1077-1083. 1

[16] D. Molodtsov, Soft set theory - first results, Global optimization, control, and games, III, Comput. Math. Appl., 37 (1999), 19-31. 1, 2.1

[17] D.-W. Pei, D.-Q. Miao, From soft sets to information systems, IEEE International Conference on Granular Computing, Beijing, China, 2 (2005), 617-621. 1

[18] M. Shabir, M. Naz, On soft topological spaces, Comput. Math. Appl., 61 (2011), 1786-1799. 1, 2.7, $2.9,1$

[19] Ş. Yüksel, N. Tozlu, Z. G. Ergül, Soft regular generalized closed sets in soft topological spaces, Int. J. Math. Anal. (Ruse), 8 (2014), 355-367. 1, 2.14

[20] Y. Yumak, A. K. Kaymakc1, Soft $\beta$-open sets and their applications, J. New Theory, 4 (2015), 80-89. 2.12, 2.19

[21] İ. Zorlutuna, H. Çakır, On continuity of soft mappings, Appl. Math. Inf. Sci., 9 (2015), 403-409. 1

[22] Y. Zou, Z. Xiao, Data analysis approaches of soft sets under incomplete information, Knowl.-Based Syst., 21 (2008), 941-945. 1 